\title{
DECAY WITH A RATE FOR NONCOMPACTLY SUPPORTED SOLUTIONS OF CONSERVATION LAWS
}

\author{
BLAKE TEMPLE ${ }^{1}$
}

\begin{abstract}
We show that solutions of the Cauchy problem for systems of two conservation laws decay in the supnorm at a rate that depends only on the $L^{1}$ norm of the initial data. This implies that the dissipation due to increasing entropy dominates the nonlinearities in the problem at a rate depending only on the $L^{1}$ norm of the initial data. Our results apply to any BV initial data satisfying $u_{0}( \pm \infty)=0$ and $\operatorname{Sup}\left\{u_{0}(\cdot)\right\} \ll 1$. The problem of decay with a rate independent of the support of the initial data is central to the issue of continuous dependence in systems of conservation laws because of the scale invariance of the equations. Indeed, our result implies that the constant state is stable with respect to perturbations in $L_{\text {loc }}^{1}$. This is the first stability result in an $L^{p}$ norm for systems of conservation laws. It is crucial that we estimate decay in the supnorm since the total variation does not decay at a rate independent of the support of the initial data.

The main estimate requires an analysis of approximate characteristics for its proof. A general framework is developed for the study of approximate characteristics, and the main estimate is obtained for an arbitrary number of equations.
\end{abstract}

1. Introduction. Consider the Cauchy problem for a system of $n$ conservation laws

$$
u_{t}+f(u)_{x}=0, \quad u(x, 0)=u_{0}(x),
$$

where $u \equiv\left(u_{1}, \ldots, u_{n}\right), x \in \mathbf{R}$ and $t \in \mathbf{R}^{+}$. We study decay and continuous dependence in solutions of (1), which are obtained as limits of approximate solutions generated by the random choice method of Glimm [6]. Thus we are interested in solutions that take values in a neighborhood $U$ of some constant state $\bar{u}$. We assume that $d f$, the matrix derivative of $f$, is smooth, has real and distinct eigenvalues $\lambda_{1}<\lambda_{2}<\cdots<\lambda_{n}$ in $U$, and that $\nabla \lambda_{p} \cdot R_{p}>0$ in $U$ in [9]. Here $R_{p}$ denotes the unit right eigenvector corresponding to the eigenvalue $\lambda_{p}$. By changing the frame or translating the flux function $f$ if necessary, we assume without loss of generality that $\bar{u}=0$ and $\lambda_{p}>0, p=1, \ldots, n$.

Let $u(x, t)$ denote a weak solution of (1), which is a limit of approximate solutions generated by the random choice method. The main result of this paper is the following theorem, which is proved in the case $n=2$ :

Received by the editors August 22, 1985.

1980 Mathematics Subject Classification. Primary 65M10, 76N99, 35L65, 35L67.

Key words and phrases. Riemann problem, random choice method, decay, stability, continuous dependence, conservation laws, Cauchy problem

${ }^{1}$ Sponsored by the United States Army under contract No. DAAG29-80-C-0041. This material is based upon work supported by the National Science Foundation under Grant. No. DMS-8210950, Mod. 1. 
Theorem (1). For every $V>1$ and $0<\sigma<1$ there exist constants $\delta=\delta(V)<1$ and $C(\sigma)>1$ such that, if $u_{0}(\cdot)$ satisfies

$$
\begin{gathered}
u_{0}( \pm \infty)=0, \\
T V\left\{u_{0}(\cdot)\right\}<V,
\end{gathered}
$$

and

$$
\left\|u_{0}(\cdot)\right\|_{S}<\delta
$$

then

$$
\|u(\cdot, t)\|_{S} \leqslant C(\sigma)\left\{\log \left[\frac{t}{\left\|u_{0}(\cdot)\right\|_{L^{1}}}\right]\right\}^{-1 /(2+\sigma)}
$$

for all $t>\left\|u_{0}(\cdot)\right\|_{L^{1}}$. Here constants depend only on $f$ and their arguments, \|\|$_{S}$ denotes supnorm and \|\|$_{L^{1}}$ denotes $L^{1}$ norm; i.e.

$$
\left\|u_{0}(\cdot)\right\|_{S} \equiv \operatorname{Sup}_{-\infty<x<+\infty}\left|u_{0}(x)\right|, \quad\left\|u_{0}(\cdot)\right\|_{L^{1}} \equiv \int_{-\infty}^{\infty}\left|u_{0}(x)\right| d x .
$$

If we fix the initial data $u_{0}(\cdot)$ and let $t \rightarrow \infty$, then (5) gives the decay of the solution $u(\cdot, t)$ in the supnorm at a rate independent of the support of the data. Said differently, (5) verifies that the dissipation in solutions of (1) due to increasing entropy overcomes the nonlinearities in the problem at a rate depending only on the $L^{1}$-norm of the initial data (cf. [9]). If we fix $t$ and take a sequence of initial data tending to zero in $L^{1}$, then (5) gives a rate at which the supnorm at time $t$ tends to zero with the $L^{1}$ norm of the initial data. Because the values of $u$ at time $t$ have a bounded domain of dependence, (5) also gives a rate at which $u(\cdot, t)$ tends to zero in $L_{\text {loc }}^{1}$ as the initial data tends to zero in $L_{\text {loc }}^{1}$. This is the first continuous dependence result for systems in the norm $L^{1}$.

Other decay results for systems have been obtained by Glimm-Lax, DiPerna and Liu $[4,5,7,10-14]$. For these results decay is obtained by means of estimates for the decay of the total variation. In the case of nonperiodic initial data, a rate of decay in the total variation is obtained only in the presence of compactly supported data, and the rate depends on the support of the data. It is crucial in (5) that we estimate the decay in the supnorm instead of the total variation norm because simple examples show that the total variation does not decay at a rate that depends only on the $L^{1}$ norm of the initial data.

Our interest in the $L^{1}$ norm in (5) stems from an interest in the problem of stability, by which we mean the problem of the continuous dependence of solutions on the initial data. To put the issue of stability into perspective, we make the following definitions: we say that solutions of (1) are strongly stable in a norm $\|$ || if there is a constant $C>0$ such that

$$
\|u(\cdot, t)-v(\cdot, t)\| \leqslant C\|u(\cdot, 0)-v(\cdot, 0)\|
$$

for all weak solutions $u$ and $v$. We say that solutions of (1) are weakly stable in \| \| with a rate if

$$
\|u(\cdot, t)-v(\cdot, t)\| \leqslant F(\|u(\cdot, 0)-v(\cdot, 0)\|)
$$


for all weak solutions $u$ and $v$, where $F$ is a fixed function satisfying $\lim _{\xi \rightarrow \infty} F(\xi)$ $=0$. It is well known that solutions to scalar conservation laws are $L^{1}$-contractive (cf. [8]). In the language above this says that solutions of a scalar conservation law are strongly stable in $L^{1}$ with constant $C=1$. Moreover, by studying solutions containing a single shock, it is apparent that solutions of (1) are not weakly stable in the supnorm or in the total variation norm, and are not strongly stable in any $L^{p}$, $p>1$. This leaves $L^{1}$ as a leading candidate for studying stability in systems of conservation laws. Estimate (5) proves that the constant state is weakly stable in $L_{\text {loc }}^{1}$. As a further comment, in [22] it is proven that solutions to systems are not $L^{1}$-contractive relative to a constant state in any metric that is compatible with the $u$-space topology. This directly implies that there is no metric $D$ and constant $\omega>0$ for which the following Gronwall type estimate holds in any neighborhood of $\bar{u}$ :

$$
\frac{d}{d t} \int_{-\infty}^{\infty} D(u(x, t), \bar{u}) d x \leqslant \omega \int_{-\infty}^{\infty} D(u(x, 0), \bar{u}) d x
$$

Thus, (5) gives the stability of the constant state in $L_{\text {loc }}^{1}$ in a regime where a Gronwall inequality fails in some essential way. It is an open problem whether the constant state is strongly stable in $L_{\text {loc }}^{1}$.

In the case of periodic initial data, Theorem (1) holds with $u_{0}(\cdot)$ replaced by the initial data in one period. Thus (5) gives a decay in $L^{\infty}$ at a rate independent of the period. Again, for periodic initial data, the total variation does not decay at a rate depending only on the $L^{1}$ norm of the data, and for the previous decay results the rate of decay in the total variation depends on the length of the period in the initial data. Our methods also give directly that periodic data decays like $(p / t)^{1 / 4}$, where $P$ is the length of a period. This, however, is not sharp in light of the $(p / t)$ decay rate obtained by Glimm and Lax [7].

We now indicate the proof of Theorem (1). Theorem (1) is obtained by estimating the decay of the quadratic functional $Q$, which was constructed by Glimm in [6]. Specifically, let $h$ denote a mesh length in $x$, and let $u^{h}(x, t)$ denote a corresponding approximate solution generated from initial data $u_{0}(\cdot)$ by the random choice method. Roughly speaking, the values of $u^{h}$ at time $t$ are obtained by approximating the actual solution by a set $\left\{\gamma_{l}\right\}$ of simple waves each of which moves at close to characteristic speed. The function $Q(t)$ is defined by

$$
Q(t) \equiv \sum\left|\gamma_{l}\right|\left|\gamma_{m}\right|
$$

where, again roughly, the sum is over all pairs of waves at time $t$ that will interact at some later time due to differences in the wave speeds. (In the words of Glimm, summed over all "approaching" waves.) Here, $\left|\gamma_{l}\right|$ in (8) denotes the strength of the wave $\gamma_{l}$ (for details see $\S \S 2$ and 3). In [6] it is proved that $Q$ is a positive decreasing function of time. Heuristically, this is because a term is lost from the sum in (8) whenever two waves cross each other in the $x t$-plane. The functional $Q$ measures the potential for interaction of waves, but contains no information regarding the time at which interactions will occur. Theorem (1) is a corollary of the following technical lemma, which is a sharp estimate for the rate at which $Q$ decreases as a function of 
the supnorm and the $L^{1}$ norm. For this lemma, assume that $n=2$ (or that there exists a coordinate system of Riemann invariants).

LEMMA (2A). Let $Q$ denote the quadratic functional associated with an arbitrary approximate solution $u^{h}$ which is generated from initial data $u_{0}(\cdot)$ that satisfies conditions (2)-(4) of Theorem (1). Then there exists a constant $G>0$ depending only on $f$ and $V$ and a mesh length $h_{0}=h_{0}(\varepsilon, M)$ such that, if

$$
\begin{gathered}
\left\|u^{h}(\cdot, 0)\right\|_{S} \geqslant 1 / M, \\
\left\|u^{h}(\cdot, 0)\right\|_{L^{1}}=\varepsilon
\end{gathered}
$$

and

$$
h \leqslant h_{0},
$$

then

$$
Q(0)-Q\left(\varepsilon(G M)^{2}\right) \geqslant 1 /(G M)^{2} .
$$

In words, (12) states that $Q$ will decrease by an amount on the order of the supnorm squared in a time that is on the order of the $L^{1}$-norm divided by the supnorm squared. For the case $n>2$, we obtain (12) under the assumption that the total variation of $u_{0}(\cdot)$ is small (cf. [6]).

LEMMA (2B). If $n>2$, then there exists $V \ll 1$ such that, if $u_{0}(\cdot)$ satisfies (2) and (3) of Theorem (1), then the conclusions of Lemma (2A) hold.

In the case of periodic initial data, Lemmas (2A) and (2B) hold with $u_{0}(\cdot)$ replaced by the initial data in each period.

The proof of Lemmas (2A) and (2B) is given in \$6. (See Theorem (6.3) for a detailed restatement of Lemmas (2A) and (2B).) The proof is quite technical and uses the theory of wave tracing. The theory of wave tracing was developed by Liu to prove that the random choice method converges weakly so long as the sample sequence is equidistributed [15]. Wave tracing is a method of keeping track of left and right states on approximate characteristics $[1,4,5,7,10,15,16]$. Previous decay results for systems use the theory of approximate characteristics, but rely on global mechanisms and do not require keeping track of left and right states. (It is important, however, to recognize that in [5], these methods are localized and decay in $Q$ is used to control decay in the total variation for noncompactly supported data. Of course, no rate can be obtained for decay in the total variation.) Here we develop the theory of wave tracing from what we believe is a simpler set of definitions and a simpler notation than has been previously given. The presentation is general, and essentially self-contained. Motivations for the constructions can be found in $[15,16]$.

We now deduce Theorem (1) from Lemma (2A) using the basic results of Glimm. The remainder of this paper is then devoted to the proof of Lemmas (2A) and (2B). We first give a precise statement of the results in [6]. (See [23] for a proof of the supnorm estimates.) 
Lemma (GL): Assume $u_{0}$ satisfies conditions (2)-(4) of Theorem (1). Then each approximate solution $u^{h}$ is defined for every $h>0$ and $t>0$ and, moreover, there exists $G_{0}>0$ such that

$$
\begin{gathered}
T V\left\{u^{h}(\cdot, t)\right\} \leqslant G_{0} T V\left\{u_{0}(\cdot)\right\}, \\
\left\|u^{h}(\cdot, t)\right\|_{S} \leqslant G_{0}\left\|u_{0}(\cdot)\right\|_{S}, \\
\left\|u^{h}\left(\cdot, t_{2}\right)-u^{h}\left(\cdot, t_{1}\right)\right\|_{L^{1}} \leqslant G_{0}\left\{h+\left|t_{2}-t_{1}\right|\right\}
\end{gathered}
$$

and

$$
Q\left(t_{2}\right)-Q\left(t_{1}\right) \leqslant 0
$$

for all $t_{1}<t_{2}$. (From here on we use $G_{0}$ to denote a generic constant that depends only on $V$ and $f$.)

In the case of arbitrary $n$, the results in [6] are that (13), (15) and (16) hold so long as $V$ is sufficiently small. The reason we can obtain (5) in the case $n=2$ and not $n>2$ is that we use (14), a result that is not known for $n>2$. (For $n>2$, one can show by our methods that there exists a sequence of times $t_{j}$ for which $\left\|u\left(\cdot, t_{j}\right)\right\|_{S} \rightarrow 0$ and (5) holds.)

So assume that Lemma (2A) and the assumptions of Theorem (1) hold. Let $M>1$ be given. We estimate the time at which $\left\|u^{h}(\cdot, t)\right\|_{S} \leqslant 1 / M$ for $h \leqslant h_{0}$. Let $G_{0}>1$ be large enough so that $Q(0) \leqslant G_{0}$. Set

$$
G_{1} \equiv G_{0} G
$$

and let

$$
N \equiv\left[\left(G_{1} M\right)^{2}\right]+1,
$$

where [ ] denotes "greatest integer in". Define the times $t_{1}, \ldots, t_{N}$ between which $Q$ decreases by an amount $\left(G_{1} M\right)^{-2}$ as follows:

$$
t_{0} \equiv 0, \quad t_{n+1} \equiv \operatorname{Sup}\left\{t>t_{n}: Q\left(t_{n}+\right)-Q(t) \leqslant 1 /\left(G_{1} M\right)^{2}\right\} .
$$

Define

$$
\varepsilon_{n} \equiv\left\|u^{h}\left(\cdot, t_{n}+\right)\right\|_{L^{1}}
$$

Let $m \leqslant N$ be that smallest integer for which

$$
t_{m+1}-t_{m}>\varepsilon_{m}\left(G_{1} M\right)^{2}
$$

if such an integer exists. Otherwise let $m=N$. Now if $t_{m}<\infty$, then Lemma (2A) implies that

$$
\left\|u^{h}\left(\cdot, t_{m}+\right)\right\|_{S} \leqslant 1 / G_{0} M
$$

To see this, note that the contrapositive of Lemma (2A) states that if $Q(0)$ $Q\left(\varepsilon(G M)^{2}\right)<1 /(G M)^{2}$, then $\left\|u^{h}(\cdot, 0)\right\|_{S}<1 / M$. If $t_{m}<\infty$, then this applies with $u^{h}(\cdot, 0)$ replaced by $u^{h}\left(\cdot, t_{m}+\right)$ and $M$ replaced by $G_{0} M$. (Note that if $m=N$ and $t_{N}<\infty$, then (21) holds in this case because $Q$ can incur no more than $N$ decreases of magnitude $(G M)^{-2}$.) Thus if $t_{m}<\infty$, then by (14) $\left\|u^{h}(\cdot, t)\right\|_{S} \leqslant 1 / M$ for all $t>t_{m}$ as stated. 
It remains only to estimate $t_{m}$. We show that

$$
t_{m} \leqslant\left(G_{2} M\right)^{\left(G_{2} M\right)^{2}} \varepsilon_{0}
$$

where $G_{2}=2 G_{0} G_{1}$. Without loss of generality, assume that $t_{1}>\varepsilon$ and $t_{1}>h$ so that (15) gives

$$
\varepsilon_{n} \leqslant G_{0} t_{n}
$$

where again we take $G_{0}$ to be generic. Thus if $n<m$,

$$
t_{n+1}-t_{n} \leqslant \varepsilon_{n}\left(G_{1} M\right)^{2} \leqslant G_{0} t_{n}\left(G_{1} M\right)^{2}
$$

so that

$$
t_{n+1} \leqslant\left\{1+G_{0}\left(G_{1} M\right)^{2}\right\} t_{n} .
$$

By induction this implies that

$$
\begin{aligned}
t_{m} & \leqslant\left\{1+G_{0}\left(G_{1} M\right)^{2}\right\}^{m-1} t_{1} \leqslant\left\{1+G_{0}\left(G_{1} M\right)^{2}\right\}^{m-1} \varepsilon_{0}\left(G_{1} M\right)^{2} \\
& \leqslant\left\{1+G_{0}\left(G_{1} M\right)^{2}\right\}^{m} \varepsilon_{0} \leqslant\left\{1+G_{0}\left(G_{1} M\right)^{2}\right\}^{N} \varepsilon_{0} \\
& \leqslant\left\{1+G_{0}\left(G_{1} M\right)^{2}\right\}^{\left(G_{1} M\right)^{2}} \varepsilon_{0} \leqslant\left(G_{2} M\right)^{\left(G_{2} M\right)^{2}} \varepsilon_{0},
\end{aligned}
$$

where $G_{2}=2 G_{0} G_{1}$. Thus we have that

$$
\left\|u^{h}(\cdot, t)\right\|_{S} \leqslant 1 / M
$$

so long as $t \geqslant\left(G_{2} M\right)^{\left(G_{2} M\right)^{2}} \varepsilon_{0}$. In particular, let $t=\left(G_{2} M\right)^{\left(G_{2} M\right)^{2}}$ and choose $0<\sigma<1$. Then

$$
\log \left[t / \varepsilon_{0}\right]=\left(G_{2} M\right)^{2} \log \left(G_{2} M\right) \leqslant(C(\sigma) M)^{2+\sigma}
$$

for some $C(\sigma)>0$. Thus

$$
C(\sigma)\left\{\log \left(t / \varepsilon_{0}\right)\right\}^{-1 /(2+\sigma)} \geqslant 1 / M \geqslant\left\|u^{h}(\cdot, t)\right\|_{S} .
$$

Now if $u$ is a limit of approximate solutions $u^{h}$ as $h \rightarrow 0$, then it follows that $\left\|u^{h}(\cdot, 0)\right\|_{L^{1}} \rightarrow\left\|u_{0}(\cdot)\right\|_{L^{1}}$, and we conclude that

$$
\|u(\cdot, t)\|_{S} \leqslant C(\sigma)\left\{\log \left[t /\left\|u_{0}(\cdot)\right\|_{L^{1}}\right]\right\}^{-1 /(2+\sigma)} .
$$

This completes the proof of Theorem (1).

In the case of periodic data, the estimate (31) is obtained in the same manner by replacing $u_{0}$ by the initial data in one period. In this case $C(\sigma)$ is independent of the period. For periodic data we can also use Lemmas (2A) and (2B) to obtain a rate of decay which depends on the period, since for periodic data,

$$
\left\|u^{h}(\cdot, t)\right\|_{L^{1}} \leqslant G_{0} P,
$$

where $P$ is the length of one period. In this case we can use (32) instead of (24) and argue as follows: if $u^{h}(\cdot, t)>1 / G_{0} M$, then Lemmas (2A) and (2B) give

$$
Q(t)-Q\left(t+G_{0} P\left(G_{1} M\right)^{2}\right) \geqslant 1 /\left(G_{1} M\right)^{2} \text {. }
$$




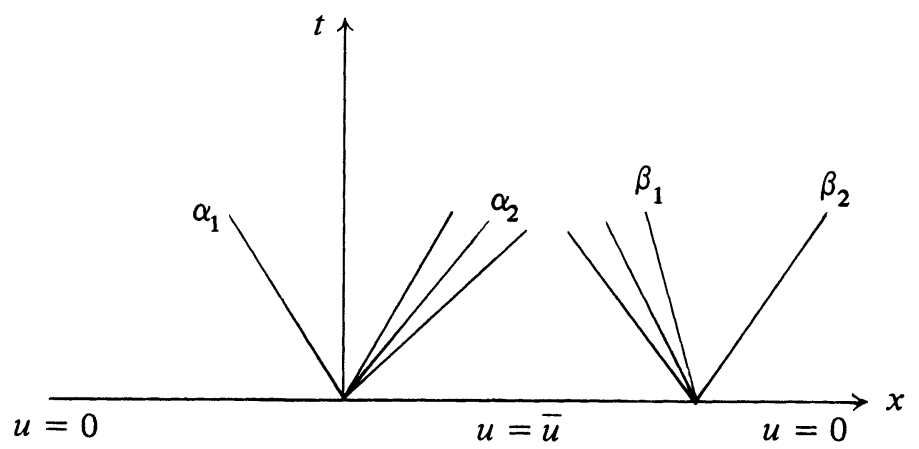

Figure 1

Since $Q(0) \leqslant G_{0}$, we see that $Q$ can incur only $G_{0}\left(G_{1} M\right)^{2}$ decreases of magnitude $1 /\left(G_{1} M\right)^{2}$ before $Q(t) \leqslant 1 /\left(G_{1} M\right)^{2}$, in which case

$$
\left\|u^{h}(\cdot, t)\right\|_{S} \leqslant 1 / G_{0} M
$$

Thus $\left\|u_{h}(\cdot, t)\right\|_{S}$ must be smaller than $1 / G_{0} M$ before time

$$
T=G_{0} P\left(G_{1} M\right)^{2} G_{0}\left(G_{1} M\right)^{2} \equiv C P M^{4} .
$$

This gives that $\left\|u^{h}(\cdot, t)\right\|_{S} \leqslant 1 / G_{0} M$ for all $t \geqslant C P M^{4}$, in which case

$$
\left\|u^{h}(\cdot, t)\right\|_{S} \leqslant C(t / p)^{-1 / 4} \text {. }
$$

We note that (33) is not sharp $\left(t^{-1}\right.$ is sharp, cf. Glimm-Lax [7]). This might be expected since we are not invoking global mechanisms of decay as in [7].

The remainder of this paper is devoted to the proof of Lemmas (2A), (2B) and (3). Before embarking on the proof, we briefly discuss the idea behind it. The idea is that, since $u_{0}( \pm \infty)=0$, if $\left\|u_{0}(\cdot)\right\|_{S}=1 / M$ and $\left\|u_{0}(\cdot)\right\|_{L^{1}}=\varepsilon$, then there must be a "spike" in the initial data of height on the order of $1 / M$ in $|u|$ and width in $x$ on the order of $\varepsilon M$. For example, if $\left|u_{0}(x)\right| \geqslant 1 / 2 M$ for all $x \in\left[x_{A}, x_{B}\right]$, then $\varepsilon=\left\|u_{0}(\cdot)\right\|_{L^{1}} \geqslant\left|x_{B}-x_{A}\right| / 2 M$, which implies $\left|x_{B}-x_{A}\right| \leqslant 2 M \varepsilon$. Thus consider the case

$$
u_{0}(x)= \begin{cases}\bar{u}, & x_{A} \leqslant x<x_{B}, \\ 0, & \text { otherwise }\end{cases}
$$

where $|\bar{u}|=1 / M, x_{B}-x_{A}=\varepsilon M$. This data resolves into four simple waves associated with the Riemann problems $[0, \bar{u}]$ and $[\bar{u}, 0]$ (cf. $[9, \S 2]$ ). Label these waves $\alpha_{1}$, $\alpha_{2}, \beta_{1}$ and $\beta_{2}$ as in Figure 1. It takes the longest time for waves to interact if $\alpha_{2}=0$ or $\beta_{1}=0$. Assume that $\alpha_{2}=0$ and to be specific, assume that $\alpha_{1}$ is a shock wave. In this case, $\beta_{1}$ must be a rarefaction wave because $u=0$ is both the leftmost and rightmost state in the problem. (Here we use the assumption that the waves are weak.) Now we can estimate the time at which $Q$ must decrease by order $1 / M^{2}$; i.e., the time it takes $\alpha_{1}$ to interact with $\beta_{1}$. But the differences in speeds between $\alpha_{1}$ and $\beta_{1}$ at time zero is on the order of $1 / M$, so interaction must occur within a time on the order of $T$, where $T$ satisfies

$$
\left|x_{B}-x_{A}\right|-T / M=0
$$


i.e., $T=M\left|x_{B}-x_{A}\right|=M^{2} \varepsilon$. Since two waves $\alpha_{1}$ and $\beta_{1}$ of strength on the order of $1 / M$ interact before time $T$, by (8) we expect $Q$ to decrease by an amount on the order $1 / M^{2}$ in time $T$. This is

$$
Q(0)-Q(T)=0(1) \frac{1}{M^{2}}
$$

which is (12).

We implement the above idea as follows: given data $u_{0}(\cdot)$, we locate $p$-waves $\alpha$ and $\beta$ whose speeds at time $t=0$ differ by $0(1) \frac{1}{M}$ and whose $x$ distance apart at $t=0$ is $0(1) \varepsilon M$ as above. We identify these waves at a later time by means of approximate characteristics. We then assume for contradiction that $Q$ does not decrease by $0(1) \frac{1}{M^{2}}$ in time $0(1) \varepsilon M^{2}$. A consequence of this is that $\alpha$ and $\beta$ may be chosen so that the corresponding characteristics do not intersect before time $0(1) \varepsilon M^{2}$. We finally derive a contradiction by estimating that since the decrease in $Q$ is small, the speed of the characteristics $\alpha$ and $\beta$ agree with the speeds at time zero sufficiently to guarantee that they intersect before time $0(1) \varepsilon M^{2}$. By this contradiction we can conclude the proof of Lemmas (2A), (2B) and (3). In the above analysis we must keep track of left and right states on approximate characteristics. This is essentially the wave tracing idea of Liu [15].

In $\$ \S 2$ and 3 we review the Riemann problem and the random choice method and establish notation. In $\$ 4$ we define approximate characteristics. In $\$ 5$ we establish properties of approximate characteristics. (This is done in a general setting.) Finally in $\S 6$ we give the proof of Lemmas (2A), (2B) and (3).

I wish to thank James Glimm for sharing with me many of his valuable insights. I would also like to thank Tai Ping Liu, Ronald J. DiPerna and I-Liang Chern for many helpful discussions.

2. The Riemann problem. The Riemann problem is the initial value problem (1) where the initial data has the form

$$
u_{0}(x)= \begin{cases}u_{L} & \text { for } x \leqslant 0 \\ u_{R} & \text { for } x>0\end{cases}
$$

We assume that the Riemann problem is uniquely solvable by the method of Lax [9] for all $u_{L}$ and $u_{R}$ in a neighborhood $U$ of $u=0$. In particular, assume that all states that appear in the solution of a Riemann problem posed in $U$ lie in a set $U_{1} \supseteq U$. Assume that $\lambda_{p}(u)<\lambda_{p^{\prime}}(v)$ for all $u, v \in U_{1}, 1<p<p^{\prime} \leqslant n$, and that (1) is genuinely nonlinear in all characteristic fields throughout $U_{1}$.

Let $\mathscr{R}_{p}\left(u_{L}\right)$ denote the integral curve of $R_{p}$ that contains the point $u_{L}$. Let $\mathscr{R}_{p}^{+}\left(u_{L}\right)$ denote the $p$-rarefaction curve associated with the point $u_{L}$; i.e., that portion of $\mathscr{R}_{p}\left(u_{L}\right)$ for which $\lambda_{p}(u) \geqslant \lambda_{p}\left(u_{L}\right)$. By [9] there exists a unique curve $\mathscr{S}_{p}\left(u_{L}\right)$ that makes $C^{2}$ piecewise $C^{3}$ contact with $\mathscr{R}_{p}\left(u_{L}\right)$ at the point $u_{L}$, and such that for each $u \in \mathscr{S}_{p}\left(u_{L}\right)$ there is a scalar $s$ such that

$$
s[u]=[f],
$$


where $[u] \equiv u-u_{L},[f]=f(u)-f\left(u_{L}\right)$. Statement (2.1) is the Rankine Hugoniot jump condition, and we say that $\mathscr{S}_{p}\left(u_{L}\right)$ is in the Hugoniot locus of $u_{L}$. Let $\mathscr{S}_{p}^{-}\left(u_{L}\right)$ denote the $p$-shock curve associated with $u_{L}$; i.e., that portion of $\mathscr{S}_{p}^{-}\left(u_{L}\right)$ for which $\lambda_{p}(u)<\lambda_{p}\left(u_{L}\right)$. We assume that $\lambda_{p}$ is monotone on both $\mathscr{S}_{p}\left(u_{L}\right)$ and $\mathscr{R}_{p}\left(u_{L}\right)$, so that the curve

$$
\mathscr{Y}_{p}\left(u_{L}\right) \equiv \mathscr{S}_{p}^{-}\left(u_{L}\right) \cup \mathscr{R}_{p}^{+}\left(u_{L}\right)
$$

is a $C^{2}$ piecewise $C^{3}$ curve throughout $U_{1}$. For $u_{R} \in \mathscr{Y}_{p}\left(u_{L}\right)$, the Riemann problem is solved by a $p$-wave: a shock wave if $\lambda_{p}\left(u_{R}\right)<\lambda_{p}\left(u_{L}\right)$ and a rarefaction wave if $\lambda_{p}\left(u_{R}\right)>\lambda_{p}\left(u_{L}\right)[9,17]$. We let $\gamma^{p}$ denote any $p$-wave. For a given $\gamma^{p}$, let $L\left(\gamma^{p}\right)$ denote the left state of $\gamma^{p}$ and let $R\left(\gamma^{p}\right)$ denote the right state. For arbitrary states $u_{L}, u_{R} \in U$, the Riemann problem is solved uniquely by $n$ waves $\gamma^{1}, \ldots, \gamma^{n}$, which are separated in the $x t$-plane (going from left to right) by the constant states $u_{i} \equiv u_{L}, \ldots, u_{n+1}=u_{R}$. The states $u_{i}$, and hence the waves $\gamma^{p}$, are uniquely determined by the condition

$$
u_{k+1} \in \mathscr{Y}_{k}\left(u_{k}\right), \quad 1 \leqslant k \leqslant n
$$

We call $\mathscr{T}_{p}$ (cf. [11]) a wave strength measure for the $p$-waves if, for each $u_{L} \in U_{1}, \mathscr{T}_{p}\left(\sigma ; u_{L}\right)$ is a parametrization of $\mathscr{Y}_{p}\left(u_{L}\right)$ in $U_{1}, \sigma \in R$, and moreover $\mathscr{T}_{p} \in C^{2}$ with bounded third derivatives with respect to both arguments, $\mathscr{T}_{p}\left(0 ; u_{L}\right)$ $=u_{L}$ and

$$
\frac{\partial \lambda_{p}\left(\mathscr{T}_{p}\left(\sigma, u_{L}\right)\right)}{\partial \sigma}>0
$$

If $L\left(\gamma^{p}\right)=u_{L}$ and $R\left(\gamma^{p}\right)=\mathscr{T}_{p}\left(\sigma ; u_{L}\right)$, then we define the signed strength of $\gamma^{p}$ to be $\sigma$. We let $\gamma^{p} \equiv \sigma$ so that " $\gamma^{p}$ " denotes both the name as well as the signed strength of the wave. By (2.3), $\gamma^{p}<0$ for shocks and $\gamma^{p}>0$ for rarefaction waves.

In the following three sections we study the random choice method approximates and associated approximate characteristics using arbitrary $\mathscr{T}$ to define wave strength. In $\S 6$ we define $\mathscr{T}$ by means of a best approximation to a coordinate system of Riemann invariants. The next lemma is a direct consequence of the fact that the speed $s$ of a shock is equal to the average characteristic speed to within terms that are quadratic in the strength of the shock $[9,11,17]$ :

Lemma (2.1). Assume that $u_{R}=\mathscr{T}_{p}\left(\sigma ; u_{L}\right), \sigma<0$. Then the speed $s$ of the corresponding shock wave satisfies $s=\lambda_{p}\left(\left(u_{L}+u_{R}\right) / 2\right)+0(1) \sigma^{2}$.

3. The random choice method approximates. In this section we define the approximate solutions of (1) generated by the random choice method of Glimm [6], and develop notation required for the subsequent sections.

Let $h$ be a mesh length in $x$, and let

$$
k=C h
$$

be the corresponding mesh length in $t, C>\operatorname{Sup}_{u \in U_{1}}\left\{\left|\lambda_{n}(u)\right|\right\}$. For $i, j \in \mathbf{Z}, j \geqslant 0$, let $x_{i} \equiv i h$ and $t_{j} \equiv j k$. Let a be a sample sequence, $\mathbf{a} \equiv\left\{a_{j}\right\}_{j=1}^{\infty}, 0<a_{j}<1$. For given initial data $u_{0}(\cdot) \subset U$, define the random choice method approximate solution $u^{h}(x, t) \equiv u^{h}\left(x, t ;\right.$ a) by induction on $j$ as follows: First, for $x_{i} \leqslant x<x_{i+1}$, define

$$
u^{h}(x, 0) \equiv u_{0}^{h}(x)=u_{0}\left(x_{i}+h / 2\right) \text {. }
$$


Next, assume for induction that $u^{h}(x, t)$ has been defined for $t<t_{j}$. Define

$$
u^{h}\left(x, t_{j}\right) \equiv u^{h}\left(x_{i}+a_{j} h, t_{j}-\right)
$$

and for $t_{j}<t<t_{j+1}$, define $u^{h}(x, t)$ to be the solution of the Riemann problem posed in (3.3) at time $t_{j}$. By (3.1), $u^{h}$ is well defined so long as $u^{h}\left(x, t_{j}\right) \subseteq U$ for all $t_{j}$.

Let $u^{h}$ be any approximate solution that is well defined by the above procedure. Let $\gamma_{i j}^{p}$ denote the name as well as the signed strength of the $p$-wave that appears in the solution of the Riemann problem that is posed at $\left(x_{i}, t_{j}\right)$ in the approximate solution $u^{h}$. Define

$$
\begin{aligned}
& L\left(\gamma_{i j}^{p}\right) \equiv \text { left state of the wave } \gamma_{i j}^{p}, \\
& R\left(\gamma_{i j}^{p}\right) \equiv \text { right state of the wave } \gamma_{i j}^{p} .
\end{aligned}
$$

If $\gamma_{i j}^{p}$ is a shock wave, define

$$
s\left(\gamma_{i j}^{p}\right) \equiv \text { speed of wave } \gamma_{i j}^{p}
$$

If $\gamma_{i j}^{p}$ is a rarefaction wave, then the wave is "split" at time $t_{j+1}$ if

$$
\lambda_{p}\left(L\left(\gamma_{i j}^{p}\right)\right) k / h<a_{j}<\lambda_{p}\left(R\left(\gamma_{i j}^{p}\right)\right) k / h .
$$

In this case define $\gamma_{i j}^{p L}$ and $\gamma_{i j}^{p R}$ by

$$
\begin{aligned}
& L\left(\gamma_{i j}^{p L}\right)=L\left(\gamma_{i j}^{p}\right), \\
& R\left(\gamma_{i j}^{p L}\right)=u^{h}\left(x_{i}+a_{j+1} h, t_{j+1}-\right)=L\left(\gamma_{i j}^{p R}\right), \\
& R\left(\gamma_{i j}^{p R}\right)=R\left(\gamma_{i j}^{p}\right) .
\end{aligned}
$$

More generally, if (3.6) does not hold, then define:

$$
\begin{gathered}
\gamma_{i j}^{p L} \equiv \begin{cases}\gamma_{i j}^{p} & \text { if } a_{j} \geqslant \lambda_{p}\left(R\left(\gamma_{i j}^{p}\right)\right) k / h, \gamma_{i j}^{p}>0, \\
\gamma_{i j}^{p} & \text { if } a_{j}>s\left(\gamma_{i j}^{p}\right) k / h, \gamma_{i j}^{p}<0, \\
0 & \text { otherwise, }\end{cases} \\
\gamma_{i j}^{p R} \equiv \begin{cases}\gamma_{i j}^{p} & \text { if } a_{j} \leqslant \lambda_{p}\left(L\left(\gamma_{i j}^{p}\right)\right) k / h, \gamma_{i j}^{p}>0, \\
\gamma_{i j}^{p} & \text { if } a_{j}<s\left(\gamma_{i j}^{p}\right) k / h, \gamma_{i j}^{p}<0, \\
0 & \text { otherwise }\end{cases}
\end{gathered}
$$

(Here, for example, $\gamma_{i j}^{p L}$ is defined to be zero if the wave lies to the right of the sample point $x_{i}+a_{j} h$, and $\gamma_{i j}^{p}$ if it lies to the left.)

By construction, the waves $\gamma_{i j}^{p}$ solve the Riemann problem for $u_{L}=$ $u^{h}\left(x_{i-1}+a_{j-1} h, t_{j}\right) \equiv L\left(\gamma_{i j}^{1}\right), u_{R}=u^{h}\left(x_{i}+a_{j-1} h, t_{j}\right) \equiv R\left(\gamma_{i j}^{n}\right)$ that is posed at $\left(x_{i}, t_{j}\right)$ in the approximate solution $u^{h}(x, t)$. Because we assume that all wave speeds are positive, the waves $\gamma_{i j}^{p}$ are formed by the interaction of the waves $\gamma_{i-1, j-1}^{p R}$ with the waves $\gamma_{i, j-1}^{p L}$ at time $t_{j-1}, p=1,2$. To emphasize this, we let $\Delta_{i j}$ denote the diamond of interactions centered at $\left(x_{i}, t_{j}\right)[6,16]$; i.e., consisting of vertex points $\left(x_{i-1}+a_{j-1} h, t_{j}\right),\left(x_{i}+a_{j-1} h, t_{j}\right),\left(x_{i}, t_{j}-\frac{1}{2} k\right)$ and $\left(x_{i}, t_{j}+\frac{1}{2} k\right)$. We say that the waves $\gamma_{i j}^{p}$, which cross the upper wedge of the diamond, are formed due to the 
interaction of the waves $\gamma_{i-1, j-1}^{p R}$ and $\gamma_{i, j-1}^{p L}$, which cross the lower wedge of the diamond. We call $\gamma_{i j}^{p}$ a wave that leaves the diamond $\Delta_{i j}$, and we call the nonzero waves among $\gamma_{i-1, j-1}^{p R}$ and $\gamma_{i, j-1}^{p L}$ the waves that enter the diamond $\Delta_{i j}$.

Two waves $\gamma_{i j}^{p}$ and $\gamma_{i^{\prime} j}^{p^{\prime}}$ are said to approach at time $t_{j}$ if one of two conditions holds [6]:

$$
p<p^{\prime} \text { and } i>i^{\prime} \text {. }
$$

or

$$
p=p^{\prime} \quad \text { and either } \quad \gamma_{i j}^{p}<0 \text { or } \quad \gamma_{i^{\prime} j}^{p^{\prime}}<0 .
$$

For $t_{j} \leqslant t<t_{j+1}$, define [4]

$$
Q(t) \equiv \sum\left|\gamma_{i j}^{p}\right|\left|\gamma_{i^{\prime} j}^{p^{\prime}}\right|
$$

where the sum is over all pairs of waves $\gamma_{i j}^{P}$ and $\gamma_{i^{\prime} j}^{p^{\prime}}$ that approach at time $t_{j}$. Define

$$
D_{i j} \equiv \sum\left|\gamma_{i-1, j-1}^{p R}\right|\left|\gamma_{i, j-1}^{p^{\prime} L}\right|
$$

where the sum is over all pairs of approaching waves that enter the diamond $\Delta_{i j}$; and finally define the cancellation

$$
C_{i j}^{p} \equiv \frac{1}{2}\left\{\left|\gamma_{i-1, j-1}^{p R}\right|+\left|\gamma_{i, j-1}^{p L}\right|-\left|\gamma_{i-1, j-1}^{p R}+\gamma_{i, j-1}^{p L}\right|\right\} .
$$

Then $C_{i j}^{p}$ measures the amount of $p$-waves cancelled from both $\gamma_{i-1, j-1}^{p R}$ and $\gamma_{i, j-1}^{p L}$ at the interaction in $\Delta_{i j}$.

The following lemmas are due to Glimm [4]. Let

$$
V_{j} \equiv \sum_{i, p}\left|\gamma_{i j}^{p}\right|
$$

estimate the total variation of $u^{h}(\cdot, t)$ for $t_{j} \leqslant t<t_{j+1}$.

LEMMA (GL1). If $u^{h} \in U_{0}$, then for all $i, j$,

$$
\left|\gamma_{i j}^{p}-\gamma_{i-1, j-1}^{p R}-\gamma_{i, j-1}^{p L}\right| \leqslant G_{0} D_{i j}
$$

This immediately implies

$$
\left|\gamma_{i j}^{p}\right|=\left|\gamma_{i-1, j-1}^{p R}\right|+\left|\gamma_{i, j-1}^{p L}\right|-2 C_{i j}^{p}+0(1) D_{i j}
$$

with $|0(1)| \leqslant G_{0}$. (Again, $G_{0}$ denotes a generic constant $)$.

LEMMA (GL2). There exist a constant $V>0$ and a number $G_{0}>0$ such that if

$$
V_{0}<V
$$

then $u^{h}$ is well defined, takes values in $U$ for all time and, moreover,

$$
\begin{gathered}
V_{j}<G_{0} V_{0} \\
G_{0}^{-1} \sum_{i} D_{i j} \leqslant Q\left(t_{j}-\right)-Q\left(t_{j}+\right) \leqslant G_{0} \sum_{i} D_{i j}
\end{gathered}
$$


and

$$
\left\|u^{h}(\cdot, t)-u^{h}(\cdot, s)\right\|_{L^{1}} \leqslant G_{0}\{h+|t-s|\}
$$

for all $j, s, t>0$.

Lemma (GL3). Assume that $u_{0}( \pm \infty)=0$ and that there exists a coordinate system of Riemann invariants (e.g. $n=2$ ). Then for every $V>0$ there exists $\tilde{M}>0$ such that if

$$
V_{0} \leqslant V
$$

and

$$
\left\|u_{0}^{h}\right\|_{S} \leqslant 1 / \tilde{M}
$$

then $u^{h}$ is well defined for all $t \geqslant 0$, takes values in $U$, and (3.18)-(3.20) hold together with

$$
\left\|u^{h}(\cdot, t)\right\|_{S} \leqslant G_{0}\left\|u^{h}(\cdot, 0)\right\|_{S} .
$$

(See [23] for a proof of Lemma (GL3).)

4. Definition of approximate characteristics. In this section we define the set of approximate characteristics (heretofore referred to as "characteristics") associated with a given approximate solution $u^{h}$ and time level $t_{J}$. In the next section, we study properties of the characteristics. The procedure is as follows. We first define an index set $\tilde{\mathscr{M}}(J)$ for the timelike piecewise linear curves that connect successive mesh points $\left(x_{i}, t_{j}\right)$ in the $x t$-plane (cf. [16]). A subset $\mathscr{M}(J) \subseteq \tilde{\mathscr{M}}(J)$ corresponds to the set of characteristics. We call this the index set for the characteristics. Each element $l \in \mathscr{M}(J)$ gives the position of an "elementary wave" $\gamma_{l}$ at different time levels. The piecewise linear curves that are undefined at $t=t_{J}$ correspond to elementary waves cancelled, and those undefined at $t=0$ correspond to elementary waves created by nonlinearities. We let $\mathscr{N} \subseteq \mathscr{M}(J)$ denote the index set for all such characteristics (cf. [15]).

We define the elementary wave $\gamma_{l}$ associated with $l \in \mathscr{M}(J)$ by assigning a left state $u_{l}^{L}[t]$ and right state $u_{l}^{R}[t]$ to each time level that intersects the piecewise linear curve defined by $l$. We define $\gamma_{l}[t] \equiv\left(u_{l}^{L}[t], u_{l}^{R}[t]\right)$, we call $\Gamma(J)=\bigcup_{l \in \mathscr{M}(J)}\left\{\gamma_{l}\right\}$ the set characteristics defined for time $t_{J}$. The assignment of states to characteristic curves is done as follows: We first state three properties that the assignment should satisfy, and then we assume the properties to hold in order to define the characteristics at the induction step. Thus the characteristics are defined and the properties are verified simultaneously at the induction step.

The characteristics determine a partitioning of the waves in $u^{h}$ appearing before time $t_{J}$. It is important to estimate the "fineness" of the partition. For Liu [15], the fineness is built into the procedure by an initial partitioning of the waves. The cost of having $\mathscr{M}(J)$ as an index set is that we must estimate the fineness of the partition as a function of $J$. This together with an estimate for the speed of characteristic 
curves is given in Theorem (5.12). The remainder of $\$ 5$ is essentially devoted to obtaining estimates for the change $\left|u^{q}[t]-u^{q}[0]\right|, q=L, R$, in terms of changes in $Q$ and in terms of strengths of elementary waves assigned to characteristic curves that cross the characteristic curve of $l$ in time $\left[0, t_{J}\right]$. We now proceed with the definition of the characteristics in detail. Let

Let $\mathscr{M}\left(j^{0}, j^{1}\right)=\left\{j^{0}, j^{0}+1, \ldots, j^{1}\right\}$, where $j^{0}<j^{1}$ are in the set $\{0,1, \ldots, J\}$.

$$
\tilde{\mathscr{M}}\left(j^{0}, j^{1}\right)=\left\{l: \mathscr{M}\left(j^{0}, j^{1}\right) \rightarrow \mathbf{Z}: l(j)-l(j-1) \in\{0,1\}\right\} .
$$

For each element of $l \in \tilde{\mathscr{M}}\left(j^{0}, j^{1}\right)$, define the function

$$
x_{i}:\left[t_{j^{0}}, t_{j^{1}}\right] \rightarrow \mathbf{R}
$$

as follows:

$$
\begin{gathered}
x_{l}\left(t_{j}\right)=x_{l(j)}, \quad j^{0} \leqslant j \leqslant j^{1}, \\
x_{l}(t)=x_{l}\left(t_{j}\right)+\frac{x_{l}\left(t_{j+1}\right)-x_{l}\left(t_{j}\right)}{k}\left(t-t_{j}\right), \quad t_{j} \leqslant t \leqslant t_{j+1} .
\end{gathered}
$$

Thus each element $l \in \tilde{\mathscr{M}}\left(j^{0}, j^{1}\right)$ corresponds to a continuous, timelike, piecewise linear curve in the $x t$-plane given by the graph of $x_{l}$. Note that the graph of $x_{l}$ is defined in $\left[t_{j^{0}}, t_{j^{1}}\right]$ and connects successive mesh points; i.e., the slope of the curve is either 0 or $k / h$. If $j, t$ is outside the domain of $l, x_{l}$, then we write $l(j)=\varnothing$, $x_{l}(t)=\varnothing$, respectively.

Let

$$
\tilde{\mathscr{M}}(J)=\bigcup_{0 \leqslant j^{0} \leqslant j^{1} \leqslant J} \tilde{\mathscr{M}}\left(j^{0}, j^{1}\right) .
$$

Then for $l \in \tilde{\mathscr{M}}(J)$, define $j_{l}^{0}, j_{l}^{1}$ (the initial and final indices of $l$ ) to be the positive integers such that $l \in \tilde{M}\left(j_{l}^{0}, j_{l}^{1}\right)$. Let $t_{l}^{0} \equiv t_{j_{l}^{0}}, t_{l}^{1} \equiv t_{j_{l}}$, and note that $t_{l}^{1} \leqslant J$.

We presently define the set $\mathscr{M}(J)$ of indices for the characteristics, as well as the set $\Gamma(J)$ of characteristics, by induction on $J$. For $p=1, \ldots, n$, the set of indices for the $p$-characteristics is a set $\mathscr{M}_{p}(J) \subset \tilde{\mathscr{M}}(J)$, and $\mathscr{M}(J)=\bigcup_{p=1}^{n} \mathscr{M}_{p}(J)$, where the RHS is a disjoint union. Corresponding to each $l \in \mathscr{M}_{p}(J)$ is the characteristic

$$
\gamma_{l}=\left(u_{l}^{L}, u_{l}^{R}\right) \in \Gamma_{p}(J)
$$

each entry being a function of $t$ for $t_{l}^{0} \leqslant t<t_{l}^{1} \leqslant t_{J}$. We let $\gamma_{l}[t] \equiv\left(u_{l}^{L}[t], u_{l}^{R}[t]\right)$ denote the value of $\gamma_{l}$ at time $t$. The functions $u_{l}^{L}[t], u_{l}^{R}[t]$ (the left and right states of the characteristics $\gamma_{l}$ at time $t$ ) are constant on intervals $t_{j} \leqslant t<t_{j+1}$ for $j_{l}^{0} \leqslant j<j_{l}^{1} . \Gamma(J)$ is the disjoint union $\Gamma(J)=\bigcup_{p=1}^{n} \Gamma_{p}(J)$. For convenience we set $\gamma_{l}[t]=0$ for $t \notin\left[t_{l}^{0}, t_{l}^{1}\right)$.

Before defining $\mathscr{M}(J)$ and $\Gamma(J)$ precisely, we first list three properties (Properties (4.1), (4.2) and (4.3)) which the characteristics satisfy. Then for $p=1, \ldots, n$ we simultaneously define $\mathscr{M}_{p}(J), \Gamma_{p}(J)$ and verify Properties (4.1)-(4.3) by induction on $J$.

Property (4.1) states that each set $\mathscr{M}_{p}(J), p=1, \ldots, n$, is partially ordered and expresses the fact that characteristics of the same family never cross: 
Property (4.1). If $l_{1}, l_{2} \in \mathscr{M}_{p}(J)$, then for every

$$
j \in\left[j_{l_{1}}^{0}, j_{l_{1}}^{1}\right] \cap\left[j_{l_{2}}^{0}, j_{l_{2}}^{1}\right] \text {, }
$$

we have that either $l_{1}(j) \leqslant l_{2}(j)$ or $l_{2}(j) \leqslant l_{1}(j)$. If $\left[j_{l_{1}}^{0}, j_{l_{1}}^{1}\right] \cap\left[j_{l_{2}}^{0}, j_{l_{2}}^{1}\right] \neq \varnothing$ and $l_{1}(j) \leqslant l_{2}(j)$ for $j$ satisfying (4.4), then we say that $l_{1} \leqslant l_{2}$ (or $l_{1}$ lies to the left of $\left.l_{2}\right)$.

Property (4.2) states that the left and right states of the $p$-characteristics $l \in \mathscr{M}_{p}(J)$ that satisfy $l(j)=i, j<J$, strict inequality $J$, partition the $p$-wave curve $\mathscr{T}_{p}\left(\cdot, L\left(\gamma_{i j}^{p}\right)\right)$ between $L\left(\gamma_{i j}^{p}\right)$ and $R\left(\gamma_{i j}^{p}\right)$ :

Property (4.2). Let $i \in \mathbf{Z}, j \in[0, J-1]$ be such that $\gamma_{i j}^{p} \neq 0$. Let $\left\{l_{0}, \ldots, l_{a-1}\right\}$ be the set of $p$-characteristics $l \in \mathscr{M}_{p}(J)$ satisfying $l(j)=i$, and ordered so that $l_{k} \leqslant l_{k+1}$ in the sense of Property (4.1). Let $u_{L} \equiv L\left(\gamma_{i j}^{p}\right), u_{R} \equiv R\left(\gamma_{i j}^{p}\right)$ and let $\sigma_{R} \equiv \gamma_{i j}^{p}$ (so that, e.g., $u_{R}=\mathscr{T}_{p}\left(\sigma_{R} ; u_{L}\right)$ ). Then for $t_{j} \leqslant t<t_{j+1}$,

$$
u_{l_{0}}^{L}(t)=u_{L}, \quad u_{l_{a-1}}^{R}(t)=u_{R}
$$

and

$$
u_{l_{k-1}}^{R}(t)=u_{l_{k}}^{L}(t)=\mathscr{T}_{p}\left(\sigma_{k} ; u_{L}\right),
$$

where $\sigma_{k} \in\left(0, \sigma_{R}\right)$ and $\left|\sigma_{k+1}\right|>\left|\sigma_{k}\right|, \leqslant k \leqslant a-1$. We define

$$
\gamma_{l_{k}}(t) \equiv \sigma_{k+1}-\sigma_{k}
$$

(i.e., round brackets around $t$ to distinguish it from $\left.\gamma_{l}[t]\right)$ to be the signed strength of the characteristic $\gamma_{l_{k}}$ at time $t \in\left[t_{j}, t_{j+1}\right), 1 \leqslant k \leqslant a-1$. Moreover, for $\sigma \in(0,1)$ and $t \in\left[t_{j}, t_{j+1}\right)$, we define

$$
u_{l_{k}}^{\sigma}(t) \equiv \mathscr{T}_{p}\left(\sigma_{k}+\sigma\left(\sigma_{k+1}-\sigma_{k}\right) ; u_{L}\right)
$$

for $1 \leqslant k \leqslant a-1$.

Note that if Property (4.2) holds, then we can use (4.6) and (4.7) to determine $\gamma_{l}(t)$ and $u_{l}^{\sigma}(t)$ for all $l \in \mathscr{M}_{p}(J), t<t_{J}$. We use (4.7) to define two characteristics $\gamma_{l_{\sigma}^{L}}$ and $\gamma_{l_{\sigma}^{R}}$ corresponding to each $\sigma \in(0,1)$ and each $l \in \mathscr{M}_{p}(J)$ satisfying $l(J)=i$, as follows:

$$
\begin{gathered}
l_{\sigma}^{q}(j)= \begin{cases}l(j) & \text { if } j \leqslant J-1, \\
i & \text { if } j=J, q=L, \\
i+1 & \text { if } j=J, q=R,\end{cases} \\
\gamma_{l_{\sigma}^{L}}[t]=\left(u_{l}^{L}[t], u_{l}^{\sigma}[t]\right), \\
\gamma_{l_{\sigma}^{R}}[t]=\left(u_{l}^{\sigma}[t], u_{l}^{R}[t]\right) .
\end{gathered}
$$

(I.e., $\sigma$ determines a splitting of the characteristic $\gamma_{l}$ into a characteristic $\gamma_{l_{o}^{L}}$ of strength $\sigma \gamma_{l}(t)$ and a characteristic $\gamma_{l_{\sigma}^{R}}$ of strength $(1-\sigma) \gamma_{l}(t)$.)

Property (4.3) expresses the fact that characteristics trace nonzero elementary waves of a given family and sign:

Property (4.3). If $l \in \mathscr{M}_{p}(J)$, then the signed strength $\gamma_{l}(t)$ of the characteristic $\gamma_{l}$ is constant and nonzero in $\left[t_{l}^{0}, t_{l}^{1}\right)$. 
When Property (4.3) holds, we write

$$
\operatorname{sgn}\left\{\gamma_{l}(t)\right\} \equiv \operatorname{sgn}(l) \text {. }
$$

For convenience, we set $\gamma_{l}(t)=0$ for $t \notin\left[t_{l}^{0}, t_{l}^{1}\right)$.

We now define $\mathscr{M}_{p}(J)$ and $\Gamma_{p}(J)$ by induction on $J$. We simultaneously verify Properties (4.1)-(4.3), which are assumed in the induction step.

First assume $J=1$. Define

where $l_{i}^{q}$ is defined by

$$
\mathscr{M}_{p}(1)=\left\{l_{i}^{q}: \gamma_{i 0}^{p q} \neq 0, p=1,2, q=L, R\right\},
$$

$$
l_{i}^{q}(0)=i, \quad l_{i}^{q}(1)= \begin{cases}i & \text { if } q=L, \\ i+1 & \text { if } q=R .\end{cases}
$$

Then for $0 \leqslant t<k \equiv t_{1}$ and $\gamma_{i 0}^{p q} \neq 0$, define

$$
u_{l_{i}^{q}}^{L}(t)=L\left(\gamma_{i 0}^{p q}\right), \quad u_{l i}^{R}(t)=R\left(\gamma_{i 0}^{p q}\right) \text {. }
$$

It is easy to verify that Properties (4.1)-(4.3) hold for $\mathscr{M}_{p}(1)$ and $\Gamma_{p}(1)$.

Now assume for induction that $\mathscr{M}_{p}(J) \subseteq \mathscr{M}(J)$ has been defined, and that for every $l \in \mathscr{M}_{p}(J)$,

$$
\gamma_{l}[t]=\left(u_{l}^{L}[t], u_{l}^{R}[t]\right)
$$

has been defined in such a way that Properties (4.1)-(4.3) are satisfied. We define $\mathscr{M}_{p}(J+1), \Gamma_{p}(J+1)$ in terms of $\mathscr{M}_{p}(J), \Gamma_{p}(J)$ and see that Properties (4.1)-(4.3) hold for $\mathscr{M}_{p}(J+1), \Gamma_{p}(J+1)$, respectively.

To define $\mathscr{M}_{p}(J+1)$ and $\Gamma_{p}(J+1)$, we define the sets

$$
\mathscr{M}_{p}^{i}(J+1)=\left\{l \in \mathscr{M}_{p}(J+1): l(J)=i\right\},
$$

for each $i \in \mathbf{Z}$. The sets $\mathscr{M}_{p}(J+1)$ and $\Gamma_{p}(J+1)$ are then defined by

$$
\begin{aligned}
\mathscr{M}_{p}(J+1) & \equiv\left\{\mathscr{M}_{p}(J) \cap \tilde{\mathscr{M}}(J-1)\right\} \cup\left\{\bigcup_{i} \mathscr{M}_{p}^{i}(J+1)\right\}, \\
\Gamma_{p}(J+1) & \equiv\left\{\gamma_{l}: l \in \mathscr{M}_{p}(J+1)\right\} .
\end{aligned}
$$

So fix $i \in \mathbf{Z}$. It remains to define $\mathscr{M}_{p}^{i}(J+1)$ and $\left\{\gamma_{l}: l \in \mathscr{M}_{p}(J+1)\right\}$. If $\gamma_{i J}^{p}=0$, then define

$$
\mathscr{M}_{p}^{i}(J+1) \equiv \mathscr{L}_{*}, \quad \Gamma_{p}^{i}(J+1) \equiv\left\{\gamma_{l}: l \in \mathscr{L}_{*}\right\}
$$

where

$$
\mathscr{L}_{*}=\left\{l \in \mathscr{M}_{p}(J): l(J)=i, \operatorname{sgn}(l) \neq \operatorname{sgn}\left(\gamma_{i J}^{p}\right)\right\} .
$$

So assume $\gamma_{i J}^{p} \neq 0$. Let $\left\{l_{0}, \ldots, l_{a-1}\right\} \subseteq \mathscr{M}_{p}(J)$ denote the indices in $\mathscr{M}_{p}(J)$ satisfying

$$
l_{k}(J)=i, \quad \operatorname{sgn}\left(l_{k}\right)=\operatorname{sgn}\left\{\gamma_{i j}^{p}\right\},
$$

and ordered so that $l_{k-1} \leqslant l_{k}, 1 \leqslant k \leqslant a-1$, in the sense of Property (4.1). Define

$$
\begin{aligned}
& u_{L} \equiv L\left(\gamma_{i J}^{p}\right), \quad u_{R} \equiv R\left(\gamma_{i J}^{p}\right), \\
& \sigma_{0}=0, \quad \sigma_{k}=\sum_{s=0}^{k-1} \gamma_{l_{s}}\left(t_{J}-\right), \quad k=1, \ldots, a, \\
& u_{k} \equiv \mathcal{T}\left(\sigma_{k} ; u_{L}\right) .
\end{aligned}
$$


Let $\sigma_{R}$ be defined for $u_{R} \equiv \mathscr{T}\left(\sigma_{R} ; u_{L}\right)$. Note that $\sigma_{k}$ is defined by $0 \leqslant k \leqslant a$, and $\gamma_{l_{k}}\left(t_{J}-\right)=\sigma_{k+1}-\sigma_{k}, k=0, \ldots, a-1$. Choose $n$ so that $\sigma_{R} \in\left(\sigma_{n}, \sigma_{n+1}\right]$. If $\left|\sigma_{R}\right|$ $>\left|\sigma_{a}\right|$, let $\sigma_{a+1} \equiv \sigma_{R}$, so that then $n=a$. Let

$$
u_{M}= \begin{cases}u_{h}\left(x_{i}+a_{J+1} h, t_{J+1}-\right) & \text { if } \gamma_{i J}^{P L} \neq 0 \text { and } \gamma_{i J}^{P R} \neq 0, \\ u_{L} & \text { if } \gamma_{i J}^{P L}=0, \\ u_{R} & \text { if } \gamma_{i J}^{P R}=0,\end{cases}
$$

and let $\sigma_{M}$ be defined by $u_{M}=\mathscr{T}\left(\sigma_{M} ; u_{L}\right)$. Now for $0 \leqslant k<n$, define

$$
\begin{aligned}
& l_{k}^{\prime}(j) \equiv \begin{cases}l_{k}(j) & \text { if } j \leqslant J, \\
i & \text { if } j=J+1,\left|\sigma_{k}\right|<\left|\sigma_{M}\right|, \\
i+1 & \text { if } j=J+1,\left|\sigma_{k}\right| \geqslant\left|\sigma_{M}\right|, \\
\varnothing & \text { if } j>J+1,\end{cases} \\
& \gamma_{l_{k}^{\prime}}[t] \equiv \begin{cases}\gamma_{l_{k}}[t] & \text { if } t<t_{J}, \\
\left(u_{k}, u_{k+1}\right) & \text { if } t_{J} \leqslant t<t_{J+1}, \\
0 & \text { if } t \geqslant t_{J+1}\end{cases}
\end{aligned}
$$

For $k=n$, define (cf. (4.8)-(4.10))

$$
\begin{gathered}
l_{n}^{L}(j)= \begin{cases}\varnothing & \text { if } j<J, n=a, \\
i & \text { if } j=J, n=a, \\
l_{n}(j) & \text { if } j \leqslant J, n<a, \\
i & \text { if } j=J+1,\left|\sigma_{n}\right|<\left|\sigma_{M}\right|, \\
i+1 & \text { if } j=J+1,\left|\sigma_{n}\right| \geqslant\left|\sigma_{M}\right|, \\
\varnothing & \text { if } j>J+1,\end{cases} \\
l_{n}^{R}(j)= \begin{cases}\varnothing & \text { if } j \leqslant J+1, n=a, \\
l_{n}(j) & \text { if } j \leqslant J, n<a, \\
i & \text { if } j=J+1,\left|\sigma_{n}\right|<\left|\sigma_{M}\right|, n<a, \\
i+1 & \text { if } j=J+1,\left|\sigma_{n}\right| \geqslant\left|\sigma_{M}\right|, n<a, \\
\varnothing & \text { if } j>J+1,\end{cases} \\
\gamma_{l_{n}^{L}}[t]= \begin{cases}0 & \text { if } t<t_{J}, n=a, \\
\left(u_{a}, u_{a+1}\right) & \text { if } t_{J} \leqslant t<t_{J+1}, n=a, \\
\gamma_{l_{n o}{ }^{L}}[t], \sigma=\frac{\sigma_{R}-\sigma_{n}}{\sigma_{n+1}-\sigma_{n}} \quad \text { if } t<t_{J}, n<a, \\
\left(u_{n}, u_{R}\right) & \text { if } t_{J} \leqslant t<t_{J+1}, n<a, \\
0 & \text { if } t \geqslant t_{J+1},\end{cases} \\
\gamma_{l_{n}^{R}}[t]= \begin{cases}0 & \text { if } t<t_{J+1}, n=a, \\
0 & \text { if } t_{J} \leqslant t, n<a, \\
\gamma_{l_{n o}{ }^{R}}[t], \sigma=\frac{\sigma_{R}-\sigma_{n}}{\sigma_{n+1}-\sigma_{n}} \quad \text { if } t<t_{J}, n<a, \\
0 & \text { if } t \geqslant t_{J+1} .\end{cases}
\end{gathered}
$$


Let

$$
\mathscr{L}_{<n} \equiv\left\{l_{k}^{\prime}: 0 \leqslant k<n\right\}, \quad \mathscr{L} \equiv\left\{l_{n}^{L}, l_{n}^{R}\right\}, \quad \mathscr{L}_{>n} \equiv\left\{l_{k}: n<k \leqslant a-1\right\} .
$$

Define

$$
\mathscr{L}^{*} \equiv\left\{l \in \mathscr{L}_{<n} \cup \mathscr{L}_{n}: \gamma_{l}\left(t_{J}+\right) \neq 0\right\}
$$

For $l \in \mathscr{L}^{*}$ let $\sigma_{l}^{q}, q=L, R$, be defined by $u q\left(t_{J}+\right)=\mathscr{T}\left(\sigma_{l}^{q} ; u_{L}\right)$, and set

$$
\mathscr{L}_{\neq M} \equiv\left\{l \in \mathscr{L}^{*}: \sigma_{M} \notin\left(\sigma_{l}^{L}, \sigma_{l}^{R}\right)\right\} .
$$

Moreover if $l \in \mathscr{L}^{*}$ and $\sigma_{M} \in\left(\sigma_{l}^{L}, \sigma_{l}^{R}\right)$, then let $\sigma \equiv\left(\sigma_{M}-\sigma_{l}^{L}\right) /\left(\sigma_{l}^{R}-\sigma_{l}^{L}\right)$, and set (cf. (4.8)-(4.10))

$$
\mathscr{L}_{M} \equiv\left\{l_{\sigma}^{q}: l \in \mathscr{L}^{*}, \sigma_{M} \in\left(\sigma_{l}^{L}, \sigma_{l}^{R}\right), q=L \text { or } R\right\} .
$$

Finally, we define

$$
\mathscr{M}_{p}^{i}(J+1) \equiv \mathscr{L}_{\neq M} \cup \mathscr{L}_{M} \cup \mathscr{L}_{>n} \cup \mathscr{L}_{*},
$$

in the case $\gamma_{i J}^{p} \neq 0$.

Since $\gamma_{l}$ is defined for all $l \in \mathscr{M}_{p}^{i}(J+1)$ this completes the definition of $\mathscr{M}_{p}^{i}(J+1)$ and $\Gamma_{p}^{i}(J+1)$. We leave it to the reader to verify from the above construction that Properties (4.1)-(4.3) are satisfied by $\mathscr{M}_{p}(J+1)$ and $\Gamma_{p}(J+1)$. This completes the definition of the approximate characteristics.

We say that $\gamma_{l} \in \Gamma(J)$ is cancelled at $\left(x_{i}, t_{j}\right)$ if $l(j)=i$ and $j=j_{l}$. From (4.16) it is clear that $\gamma_{l} \in \Gamma(J+1)$ is cancelled at $\left(x_{i}, t_{J}\right)$ if and only if $l \in \mathscr{L}_{>n} \cup \mathscr{L}_{*}$ or $l=l_{n}^{R}$ where $n=a$.

5. Properties of approximate characteristics. In this section we study properties of the characteristics $\Gamma(J)$ and index set $\mathscr{M}(J)$ defined in the previous section. Let $u^{h}$ be a given approximate solution generated by the random choice method from initial data $u_{0}$. The sets $\Gamma(J)$ and $\mathscr{M}(J)$ associated with $u^{h}$ are determined by the choices of $u_{0}, J, \mathbf{a}, h$ and $\mathscr{T}$, which we take to be given. We let $\mathscr{M} \equiv \mathscr{M}(J), \Gamma \equiv \Gamma(J)$. Throughout the remainder of the paper, $\Sigma_{\mathscr{L}}$ is taken to mean $\Sigma_{l \in \mathscr{L}}$ whenever $\mathscr{L} \subseteq \mathscr{M}$. We now develop some definitions.

Let $\mathscr{N}(t)$ denote the index set for the "null" characteristics that are either cancelled or else are created by "nonlinearities" in time $[0, t], t<t_{J}$; i.e.,

$$
\mathscr{N}(t) \equiv\left\{l \in \mathscr{M}: t_{l}^{0}>0 \text { or } t_{l}^{1} \leqslant t\right\} .
$$

We partition $\mathscr{N}(t)$ into $\mathscr{N}_{0}(t)$ and $\mathscr{N}_{\varnothing}(t)$ as follows:

$$
\begin{aligned}
& \mathcal{N}_{0}(t) \equiv\left\{l \in \mathscr{N}(t): t_{l}^{0}=0\right\}, \\
& \mathcal{N}_{\varnothing}(t) \equiv\left\{l \in \mathscr{N}(t): t_{l}^{0}>0\right\} .
\end{aligned}
$$

By Property (4.3), the index set $\mathscr{M}$ partitions into $\mathscr{M}=\mathscr{M}^{+} \cup \mathscr{M}^{-}$, where $\mathscr{M}^{+} \equiv\{l$ $\in \mathscr{M}: \operatorname{sgn}(l)>0\}$ and $\mathscr{M}^{-} \equiv\{l \in \mathscr{M}: \operatorname{sgn}(l)<0\}$. We call $\mathscr{M}^{+}\left[\right.$resp. $\left.\mathscr{M}^{-}\right]$the set of rarefaction wave [resp. shock wave] characteristics. For $p=1, \ldots, n$ and $q=+$ or -, define

$$
\mathscr{M}_{p}^{q} \equiv \mathscr{M}_{p} \cap \mathscr{M}^{q}
$$


Similarly, define $\mathcal{N}_{p}^{q}(t) \equiv \mathscr{N}(t) \cap \mathscr{M}_{p}^{q}$, and set

$$
\mathscr{N}_{p}^{q}(t) \equiv \mathscr{N}_{p}^{q}(t) \cap \mathscr{N}_{0}(t), \quad \mathscr{N}_{p \varnothing}^{q}(t) \equiv \mathscr{N}_{p}^{q}(t) \cap \mathscr{N}_{\varnothing}(t),
$$

so that $\mathscr{N}_{p}^{q}(t)$ is the disjoint union of $\mathscr{N}_{p}^{q}(t) \cup \mathcal{N}_{p \varnothing}^{q}(t)$.

We say that two characteristics $\gamma_{l}$ and $\gamma_{m}$ intersect at time $t_{j}$ if $l(j)=m(j)$ and $l(j-1) \neq m(j-1)$. The following lemma which can verified by induction on $J$ implies the uniqueness of intersection times for characteristics in different families.

Lemma (5.1). If $l \in \mathscr{M}_{p}, m \in \mathscr{M}_{p}$ and $p<p^{\prime}$, then $x_{m}-x_{l}$ is a nondecreasing function of time for $t \in\left[t_{l}^{0}, t_{l}^{1}\right] \cap\left[t_{m}^{0}, t_{m}^{1}\right]$.

The next lemma follows from Properties (4.1)-(4.3):

LeMma (5.2). If $l, m \in \mathscr{M}_{p}, \operatorname{sgn}(l) \neq \operatorname{sgn}(m)$, and l intersects $m$ at time $t_{j} \leqslant t<t_{J}$, then either $l \in \mathcal{N}(T)$ or $m \in \mathcal{N}(t)$. Moreover, if $l$ and $m$ are both shock wave characteristics, then there is at most one interesection time $t_{0}$, and for all $t \in\left[t_{0}, t_{l}^{1}\right) \cap$ $\left[t_{0}, t_{m}^{1}\right)$ we have $x_{l}(t)=x_{m}(t)$.

Define

$$
N_{i j}(l) \equiv \begin{cases}\left|\gamma_{l}\left(t_{j}+\right)\right|-\left|\gamma_{l}\left(t_{j}-\right)\right| & \text { if } l(j)=i, \\ 0 & \text { otherwise. }\end{cases}
$$

We call $N_{i j}(l)$ the nonlinearity contributed by the characteristic $\gamma_{l}$ at the mesh point $\left(x_{i}, t_{j}\right)$ (cf. [16]). In particular, since $\gamma_{l}(t)$ is constant on $\left[t_{l}^{0}, t_{l}^{1}\right]$, we must have $N_{i j}(l)=0$ unless $l \in \mathcal{N}\left(t_{j}\right)$ and either $j_{l}^{0}=j$ or $j_{l}^{1}=j$. Define

$$
\begin{aligned}
C_{i j}(l) & = \begin{cases}\left|\gamma_{l}\left(t_{j}-\right)\right| & \text { if } l(j)=i \text { and } j=j_{l}^{1}, \\
0 & \text { otherwise, }\end{cases} \\
E_{i j}(l) & = \begin{cases}\left|\gamma_{l}\left(t_{j}+\right)\right| & \text { if } l(j)=i \text { and } j=j_{l}^{0}, \\
0 & \text { otherwise, }\end{cases}
\end{aligned}
$$

so that

$$
N_{i j}(l)=-C_{i j}(l)+E_{i j}(l) .
$$

We call $C_{i j}(l)$ the cancellation and $E_{i j}(l)$ the error in the nonlinearity $N_{i j}(l)$. The following proposition is a restatement of Lemma (GL1) in the language of approximate characteristics:

Proposition (5.3). There exists $G_{0}>0$ such that

$$
\sum_{\mathscr{M}} E_{i j}(l) \leqslant G_{0} D_{i j}
$$

and

$$
\left|\left\{\sum_{\mathcal{M}_{p}^{q}} C_{i j}(l)\right\}-C_{i j}^{p}\right| \leqslant G_{0} D_{i j}
$$

for $p=1, \ldots, n$ and $q=+$ or - . 
(Note that since $E_{i j}(l)=C_{i j}(l)=0$ for $l \notin \mathscr{N}\left(t_{j}\right)$, sums over $\mathscr{M}$ and $\mathscr{M}_{p}^{q}$ can be replaced by sums over $\mathscr{N}\left(t_{j}\right)$ and $\mathscr{N}_{p}^{q}\left(t_{j}\right)$ in (5.10) and (5.11), respectively.)

COROLlaRY (5.3). There exists $G_{0}>0$ such that

$$
\begin{gathered}
\sum_{\mathcal{N}_{\varnothing}(t)}\left|\gamma_{l}\right| \leqslant G_{0}[Q(0)-Q(t)], \\
\sum_{\mathscr{M}}\left|\gamma_{l}\right| \leqslant G_{0} V_{0} .
\end{gathered}
$$

Proof. If $l \in \mathscr{N}_{\varnothing}(t)$, then $t_{l}^{0}>0$. Thus by (5.5),

$$
\left|\gamma_{l}\right|=E_{l\left(j_{l}^{0}\right), j_{l}^{0}}(l)
$$

and so by Lemma (GL1)

$$
\sum_{\mathscr{N}_{\varnothing}(t)}\left|\gamma_{l}\right| \leqslant \sum_{i j} \sum_{\mathscr{M}} E_{i j}(l) \leqslant \sum_{i j} G_{0} D_{i j} \leqslant G_{0}[Q(0)-Q(t)] .
$$

This verifies (5.12A). For (5.12B), note that since $\left|\gamma_{l}(t)\right|$ is constant on $\left[t_{l}^{0}, t_{l}^{1}\right)$, we must have

$$
\sum_{\mathscr{M} \backslash \mathscr{N}_{\varnothing}\left(t_{J}\right)}\left|\gamma_{l}\right| \leqslant G_{0} V_{0}
$$

Thus by (5.12A)

$$
\sum_{\mathscr{M}}\left|\gamma_{l}\right|=\sum_{\mathscr{M} \backslash \mathcal{N}_{\varnothing}\left(t_{J}\right)}\left|\gamma_{l}\right|+\sum_{\mathcal{N}_{\varnothing}\left(t_{J}\right)}\left|\gamma_{l}\right| \leqslant G_{0} V_{0} .
$$

For $j \leqslant J$, define the set of indice pairs associated with waves that approach at time $t$ (cf. (3.11)): $\mathscr{A}(t)=\mathscr{A}^{1}(t) \cup \mathscr{A}^{2}(t)$, where

$$
\begin{array}{r}
\mathscr{A}^{1}(t) \equiv\left\{\langle l, m\rangle: l, m \in \mathscr{M}_{p} \text { for some } p=1, \ldots, n, x_{l}(t)<x_{m}(t),\right. \\
\left.\quad \text { and either } l \text { or } m \text { is in } \mathscr{M}^{-}\right\},
\end{array}
$$

$$
\mathscr{A}^{2}(t) \equiv\left\{\langle l, m\rangle: l \in \mathscr{M}_{p}, m \in \mathscr{M}_{p^{\prime}} \text { for } p<p^{\prime} \text {, and } x_{l}(t)>x_{m}(t)\right\} .
$$

(Here we use $\langle l, m\rangle$ to denote the set or unordered pair $\{l, m\}$. Note also that $x_{l}(t)<x_{m}(t)$ implies $x_{l}(t) \neq \varnothing \neq x_{m}(t)$, so that $\left|\gamma_{l}(t)\right| \neq 0 \neq\left|\gamma_{m}(t)\right|$.)

We call $\mathscr{A}^{1}(t)$ and $\mathscr{A}^{2}(t)$ the index sets for the characteristics that approach at time $t$ and are in the same and different families, respectively. Define

$$
\mathscr{A}_{i j} \equiv\left\{\langle l, m\rangle: l(j)=m(j)=i \text { and }\langle l, m\rangle \in \mathscr{A}\left(t_{j}-\right)\right\} .
$$

The following is a consequence of Property (4.3) together with the definition of $Q$ and $D_{i j}$ :

LEMMA (5.4). We have

$$
Q(t)=\sum_{\langle l, m\rangle \in \mathscr{A}(t)}\left|\gamma_{l}\right|\left|\gamma_{m}\right|
$$


and

$$
D_{i j}=\sum_{\langle l, m\rangle \in \mathscr{A}_{i j}}\left|\gamma_{l}\right|\left|\gamma_{m}\right|
$$

From here on out, we write $\Sigma_{\mathscr{A}}$ in place of $\Sigma_{\langle l, m\rangle \in \mathscr{A}}$, etc.

Define $\mathscr{A}_{0}(t)$ and $\mathscr{A}_{\varnothing}(t)$ as follows:

$$
\begin{aligned}
& \mathscr{A}_{0}(t) \equiv\left\{\langle l, m\rangle \in \mathscr{A}(0): \text { either lor } m \text { is in } \mathscr{N}_{0}(t)\right\} \\
& \mathscr{A}_{\varnothing}(t) \equiv\left\{\langle l, m\rangle \in \mathscr{A}(t): \text { either lor } m \text { is in } \mathscr{N}_{\varnothing}(t)\right\} .
\end{aligned}
$$

Lemma (5.5). Let $\mathscr{A}^{\prime} \subseteq \mathscr{A}(0) \backslash \mathscr{A}(T), T<t_{J}$. Then

$$
\mathscr{A}(T) \subseteq \mathscr{A}(0) \cup \mathscr{A}_{\varnothing}(t) \backslash \mathscr{A}^{\prime} \text {. }
$$

Proof. By the definition of $\mathscr{A}(t),\langle l, m\rangle \in \mathscr{A}(t)$ implies that $\gamma_{l}(t) \neq 0$ and $\gamma_{m}(t) \neq 0$, thus $\mathscr{A}(T) \cap \mathscr{A}^{\prime}=\varnothing$. Assume then that $l$ and $m$ are in $\mathscr{M} \backslash \mathscr{N}_{\varnothing}(T)$ and $\langle l, m\rangle \in \mathscr{A}(T)$. It remains only to show that $\langle l, m\rangle \in \mathscr{A}(0)$. But by the definition of $\mathscr{A}$, the only way $\langle l, m\rangle$ could fail to be in $\mathscr{A}(0)$ is if $l$ and $m$ intersect at two distinct times in [0,T], which contradicts Lemma (5.1) or (5.2). For example, if $l \in \mathscr{M}_{p}$ and $m \in \mathscr{M}_{p^{\prime}}, p<p^{\prime}$, then by (5.14), $x_{l}(T)>x_{m}(T)$. Thus by Lemma (5.1), $x_{l}(t)>x_{m}(t)$ for all $t<T$. Hence $\langle l, m\rangle \in \mathscr{A}(0)$.

Lemma (5.6). There exists $G_{0}>0$ such that, for all $T<t_{J}$,

$$
\sum_{\mathscr{A}_{\varnothing}(T)}\left|\gamma_{l}\right|\left|\gamma_{m}\right| \leqslant G_{0}[Q(0)-Q(T)]
$$

Proof. By (5.12) and (5.13),

$$
\sum_{\mathscr{A}_{\varnothing}(T)}\left|\gamma_{l}\right|\left|\gamma_{m}\right| \leqslant \sum_{l \in \mathscr{M}}\left|\gamma_{l}\right| \sum_{m \in \mathcal{N}_{\varnothing}}\left|\gamma_{m}\right| \leqslant G_{0}[Q(t)-Q(0)] .
$$

LEMMA (5.7). There exists $G_{0}>0$ such that, if

$$
\mathscr{A}^{\prime} \subseteq \mathscr{A}(0) \backslash \mathscr{A}(T), \quad T<t_{J},
$$

and

$$
\sum_{\mathscr{A}^{\prime}}\left|\gamma_{l}\right|\left|\gamma_{m}\right| \geqslant L
$$

then

$$
Q(0)-Q(T) \geqslant L / G_{0}
$$

Proof. By Lemma (5.5) and (5.22) we have

$$
\mathscr{A}(T) \subseteq \mathscr{A}(0) \cup \mathscr{A}_{\varnothing}(T) \backslash \mathscr{A}^{\prime} .
$$

Thus

$$
\begin{aligned}
Q(T) & =\sum_{\mathscr{A}(T)}\left|\gamma_{l}\right|\left|\gamma_{m}\right| \leqslant \sum_{\mathscr{A}(0)}\left|\gamma_{l}\right|\left|\gamma_{m}\right|+\sum_{\mathscr{Q}(T)}\left|\gamma_{l}\right|\left|\gamma_{m}\right|-\sum_{\mathscr{A}^{\prime}}\left|\gamma_{l} \| \gamma_{m}\right| \\
& \leqslant Q(0)+G_{0}[Q(0)-Q(T)]-L .
\end{aligned}
$$


Combining terms, this gives $Q(0)-Q(T) \geqslant L /\left(G_{0}+1\right)$. Since $G_{0}$ is generic, this verifies (5.24).

Proposition (5.8). There exists $G_{0}>0$ such that, if

$$
\sum_{\mathscr{N}(T)}\left|\gamma_{l}\right| \geqslant L
$$

for $T<t_{J}$, then

$$
Q(0)-Q(T) \geqslant L^{2} / G_{0} .
$$

Since $T$ is fixed here, we set $\mathscr{N}_{p}^{q} \equiv \mathscr{N}_{p}^{q}(T)$, etc.

Proof. Assume that (5.25) holds. Then for some $p \in\{1, \ldots, n\}$ and $q=+$ or we must have

$$
\sum_{\mathcal{N}_{p}^{q}(t)}\left|\gamma_{l}\right| \geqslant L / 2 n
$$

For convenience, assume $q=+$. By (5.7) and (5.8),

$$
\begin{aligned}
\sum_{\mathcal{N}_{p}^{+}(t)}\left|\gamma_{l}\right| & \leqslant \sum_{i j} \sum_{\mathcal{N}_{p}^{+}(t)} E_{i j}(l)+\sum_{i j} \sum_{\mathscr{N}_{p}^{+}(t)} C_{i j}(l) \\
& \leqslant \sum_{i j} G_{0} D_{i j}+\sum_{i j} \sum_{\mathscr{N}_{p}^{+}(t)} C_{i j}(l)
\end{aligned}
$$

where the sum on $i, j$ is over $\left\{(i, j):-\infty<i<+\infty, t_{j} \leqslant t\right\}$. But $\sum_{i j} D_{i j} \leqslant$ $G_{0}[Q(0)-Q(t)]$, together with (5.27) and (5.29), implies that for some $G_{0}>0$,

$$
\sum_{i j} \sum_{\mathscr{N}_{p}^{+}(t)} C_{i j}(l) \geqslant L / 2 n-G_{0}[Q(0)-Q(t)]
$$

and so by (5.11)

$$
\sum_{i j} C_{i j}^{p} \geqslant L / 2 n-G_{0}[Q(0)-Q(t)] .
$$

Thus we can apply (5.11) again with $q=-$ to obtain

$$
\sum_{i j} \sum_{\mathcal{N}_{p}^{-}(t)} C_{i j}(l)-\sum_{i j} C_{i j}^{p} \leqslant G_{0}[Q(0)-Q(t)],
$$

or

$$
\sum_{i j} \sum_{\mathcal{N}_{p}^{+}(t)} C_{i j}(l) \geqslant L / 2 n-G_{0}[Q(0)-Q(t)] .
$$

Therefore, by (5.7) and (5.8),

$$
\begin{aligned}
\sum_{s \in \mathscr{N}_{p}^{+}(t)}\left|\gamma_{s}\right| & \geqslant \sum_{i j} \sum_{s \in \mathscr{N}_{p}^{-}(t)} C_{i j}(s)-\sum_{i j} \sum_{s \in \mathscr{N}_{p}^{-}(t)} E_{i j}(s) \\
& \geqslant L / 2 n-G_{0}[Q(0)-Q(t)] .
\end{aligned}
$$

We now have that for some $G_{0}>0$, both

$$
\sum_{r \in \mathscr{N}_{p}^{+}}\left|\gamma_{r}\right| \geqslant L / 2 n-G_{0}[Q(0)-Q(t)]
$$


and

$$
\sum_{s \in \mathscr{N}_{p}^{+}}\left|\gamma_{s}\right| \geqslant L / 2 n-G_{0}[Q(0)-Q(t)] .
$$

By Corollary (5.3), this implies that for some $G_{0}>0, q=+$ and - , we have

$$
\sum_{\mathcal{N}_{p} q}\left|\gamma_{l}\right| \geqslant L / 2 n-G_{0}[Q(0)-Q(t)]
$$

Let $\mathscr{A}^{\prime} \equiv\left\{\langle r, s\rangle: r \in \mathscr{N}_{p 0}^{+}, s \in \mathcal{N}_{p 0}^{-}\right\}$. Then by (5.2), (5.5) and the definition of $\mathscr{A}$, $\mathscr{A}^{\prime} \subseteq \mathscr{A}(0) \backslash \mathscr{A}(T)$. Moreover,

$$
\begin{aligned}
\sum_{\mathscr{A}^{\prime}}\left|\gamma_{l}\right|\left|\gamma_{m}\right| & \geqslant \sum_{r \in \mathscr{N}_{p 0}^{+}} \sum_{s \in \mathscr{N}_{p 0}^{-0}}\left|\gamma_{r}\right|\left|\gamma_{s}\right| \geqslant\left\{L / 2 n-G_{0}[Q(0)-Q(t)]\right\}^{2} \\
& \geqslant L^{2} / 4 n^{2}-G_{0}[Q(0)-Q(t)]
\end{aligned}
$$

where we use the fact that $L \leqslant V_{0} \leqslant G_{0}$ and $[Q(0)-Q(t)] \leqslant G_{0}$ for some $G_{0}>0$. Thus by Lemma (5.7),

$$
Q(0)-Q(T) \geqslant L^{2} / G_{0} 4 n^{2}-[Q(0)-Q(T)],
$$

or

$$
Q(0)-Q(T) \geqslant L^{2} / 8 G_{0} n^{2}
$$

Since $G_{0}$ is generic we can take $1 / 8 G_{0} n^{2}$ to be $G_{0}$, so (5.35) establishes Proposition (5.8).

COROLlaRY (5.8). There exists $G_{0}$ positive so that if $T<t_{J}$ and

$$
Q(0)-Q(T) \leqslant L
$$

then

$$
\sum_{\mathscr{N}(T)}\left|\gamma_{l}\right| \leqslant G_{0} L^{1 / 2}
$$

We next define index sets for characteristics that intersect a given characteristic in a given time interval. Let

$$
\begin{aligned}
\mathscr{B}_{l}(j) \equiv\{m \in \mathscr{M}: m(j) & \left.=l(j) \text { and }\langle l, m\rangle \in \mathscr{A}^{2}\left(t_{j}-\right)\right\}, \\
B_{l}(j) & \equiv \sum_{\mathscr{B}_{l}(j)}\left|\gamma_{m}\right|,
\end{aligned}
$$

and for $t_{1} \in\left[t_{j 1-1}, t_{j 1}\right)$ and $t_{2} \in\left[t_{j 2}, t_{j 2+1}\right)$, let

$$
B_{l}\left[t_{1}, t_{2}\right] \equiv \sum_{j 1}^{j 2} B_{l}(j)
$$

In addition, for $l \in \mathscr{M}_{p}^{-}$, define

$$
\begin{aligned}
\mathscr{B}_{l R}^{-}(j) & \equiv\left\{m \in \mathscr{M}_{p}^{-}: m(j)=l(j), m(j-1)>l(j-1)\right\}, \\
\mathscr{B}_{l L}^{-}(j) & \equiv\left\{m \in \mathscr{M}_{p}^{-}: m(j)=l(j), m(j-1)<l(j-1)\right\}, \\
\mathscr{B}_{l}^{-}(j) & =\mathscr{B}_{l R}^{-}(j) \cup \mathscr{B}_{l L}^{-}(j) .
\end{aligned}
$$


For $q=L, R$ or absent, define $\mathscr{B}_{l q}^{-}\left[t_{1}, t_{2}\right]$ as in (5.40). (Note here that script $\mathscr{B}$ denotes a set, while upper case $B$ denotes a real number.)

In the next section, we use the following technical result:

Proposition (5.9). Let

$$
\mathscr{M}_{p}^{-}\left[i_{L}, i_{R}\right] \equiv\left\{l \in \mathscr{M}_{p}^{-}: l(0) \in\left[i_{L}, i_{R}\right]\right\} .
$$

Assume that $s \in \mathscr{M}_{p}^{-} \backslash \mathscr{N}(T), s(0)=i_{L}$, that

$$
\sum_{\mathscr{M}_{p}^{-}\left[i_{L}, i_{R}\right]}\left|\gamma_{l}\right| \geqslant 4 L
$$

and that

$$
\left|\gamma_{i 0}^{p}\right| \leqslant L
$$

for all $i \in\left[i_{L}, i_{R}\right]$ such that $\gamma_{i 0}^{p}<0$. Then there exists $G_{0}>0$ such that, if $L<\mu$ and

$$
\sum_{\mathscr{B}_{p R}^{-}[0, T]}\left|\gamma_{l}\right|>L
$$

Then

$$
Q(0)-Q(T) \geqslant G_{0}^{-1} L^{2}
$$

Moreover, if the hypotheses are satisfied with $s(0)=i_{R}$ and $\mathscr{B}_{p L}^{-}[0, T]>L$, then again (5.45) follows.

To prove Proposition (5.9), we use following lemma:

LEMMA (5.9). Let $\left\{L_{i}\right\}_{i=1}^{m}$ be a nonnegative sequence of numbers which satisfies

$$
\begin{aligned}
& 0 \leqslant L_{i} \leqslant L, \\
& \sum_{i=1}^{m} L_{i} \geqslant 3 L .
\end{aligned}
$$

Then

$$
\sum_{1 \leqslant i<j \leqslant m} L_{i} L_{j} \geqslant L^{2}
$$

Proof. Let $i^{*}=\inf \left\{i: \sum_{i=1}^{i^{*}} L_{i} \geqslant L\right\}$. Then $L_{i^{*}} \leqslant L$ implies that $\sum_{i=i^{*}+1} L_{i} \geqslant L$. Therefore,

$$
\sum_{1 \leqslant i<j \leqslant m} L_{i} L_{j} \geqslant\left(\sum_{i=1}^{i^{*}} L_{i}\right)\left(\sum_{i=i^{*}+1}^{m} L_{i}\right) \geqslant L^{2} .
$$

Proof of Proposition (5.9). Let $\mathscr{B}_{0} \equiv \mathscr{B}_{p R}^{-}[0, T] \backslash \mathscr{N}(T)$ and $\mathscr{B}_{\varnothing} \equiv \mathscr{B}_{p R}^{-}[0, T]$ $\cap \mathscr{N}(T)$. Let $L_{0}=\sum_{\mathscr{B}_{0}}\left|\gamma_{l}\right|$ and $L_{\varnothing}=\sum_{\mathscr{B}_{\varnothing}}\left|\gamma_{l}\right|$. Without loss of generality, assume $L_{0}>3 L$. If not, then $L_{\varnothing}>L$, in which case Proposition (5.8) implies that

$$
Q(0)-Q(T) \geqslant L^{2} / G_{0},
$$

which gives (5.45). Let $\mathscr{B}_{i 0} \equiv\left\{l \in \mathscr{B}_{0}: l(0)=i\right\}$, and define $L_{i}=\sum_{\mathscr{B}_{i 0}}\left|\gamma_{l}\right|$.

Let $i^{\prime \prime}=\max \left\{i: L_{i} \neq 0\right\}$, and let $i^{\prime}=\min \left\{i^{\prime \prime}, i_{R}\right\}$. By (5.42) and (5.44),

$$
\sum_{i=i_{L}}^{i^{\prime \prime}} L_{i} \geqslant 3 L \text {. }
$$


Moreover by Property (4.3), $L_{i}=-\gamma_{i 0}^{p}$ if $\gamma_{i 0}^{p}<0$ and $L_{i}=0$ otherwise, so that $L_{i} \leqslant L$ for all $i \in\left[i_{L}, i^{\prime \prime}\right]$. Thus the conditions of Lemma (5.9) hold, and we have

$$
\sum_{i_{L} \leqslant i<j \leqslant i^{\prime}} L_{i} L_{j} \geqslant L^{2}
$$

But by definition, $\langle l, m\rangle \in \mathscr{A}^{\prime}(0) \backslash \mathscr{A}^{\prime}(T)$ if $l, m \in \mathscr{B}_{0}$ and $l(0) \neq m(0)$. Thus

$$
\mathscr{A}(0) \backslash \mathscr{A}(T) \geqslant \sum_{\mathscr{A}^{\prime}}\left|\gamma_{l}\right|\left|\gamma_{m}\right| \geqslant \sum_{i_{L} \leqslant i<j \leqslant i^{\prime}} L_{i} L_{j} \geqslant L^{2} .
$$

Therefore, by Lemma (5.7) we conclude

$$
Q(0)-Q(T) \geqslant L^{2} / G_{0} .
$$

Finally, define $\lambda_{l}[t]$, the speed of the characteristic $\gamma_{l}$ at time $t$ as follows: Let $l \in \mathscr{M}_{p}, t \in\left[t_{j}, t_{j+1}\right) \cap\left[t_{l}^{0}, t_{l}^{1}\right)$, and assume that $l(j)=i$. Then

$$
\lambda_{l}[t] \equiv \begin{cases}\lambda\left(\gamma_{i j}^{p}\right) & \text { if } l<0 \\ \lambda_{p}\left(u_{l}^{L}[t]\right) & \text { if } l>0\end{cases}
$$

We now estimate the change in $u_{l}^{L}, u_{l}^{R}$ and $\lambda_{l}$ for $l \in \mathscr{M}(J) \backslash \mathscr{N}(J)$.

Lemma (5.10). Let $l \in \mathscr{M}(J) \backslash \mathscr{N}(J)$. Then

$$
\begin{gathered}
\left|u_{l}^{q}\left[t_{j}+\right]-u_{l}^{q}\left[t_{j}-\right]\right| \leqslant G_{0}\left\{B_{l}(j)+D_{l(j), j}\right\}, \\
\left|\lambda_{l}\left[t_{j}+\right]-\lambda_{l}\left[t_{j}-\right]\right| \leqslant G_{0}\left\{B_{l}(j)+B_{l}^{-}(j)+C_{l(j), j}+D_{l(j), j}\right\}
\end{gathered}
$$

for $q=L, R$.

Corollary (5.10). Let $l \in \mathscr{M} \backslash \mathscr{N}(T), t_{1}<t_{2}<T<t_{J}$. Then for $q=L$ and $R$,

$$
\begin{gathered}
\left|u_{l}^{q}\left[t_{2}\right]-u_{l}^{q}\left[t_{1}\right]\right| \leqslant G_{0}\left\{B_{l}\left[t_{1}, t_{2}\right]+\left[Q\left(t_{1}\right)-Q\left(t_{2}\right)\right]\right\}, \\
\left|\lambda_{l}\left[t_{2}\right]-\lambda_{l}\left[t_{1}\right]\right| \leqslant G_{0}\left\{B_{l}\left[t_{1}, t_{2}\right]+B_{l}^{-}\left[t_{1}, t_{2}\right]+\left[Q\left(t_{1}\right)-Q\left(t_{2}\right)\right]^{1 / 2}\right\} .
\end{gathered}
$$

Moreover,

$$
\begin{gathered}
\left|u_{l}^{q}\left[t_{2}\right]-u_{l}^{q}\left[t_{1}\right]\right| \leqslant G_{0} V_{0}, \\
\left|\lambda_{l}\left[t_{2}\right]-\lambda_{l}\left[t_{1}\right]\right| \leqslant G_{0} V_{0} .
\end{gathered}
$$

Let a be a fixed equidistributed sequence, $\dot{0} \leqslant a_{j} \leqslant 1[15,16]$. Let $I=(c, d) \subset$ $[0,1]$ and let $0<N_{1}<N_{2}$. Define

$$
N\left(I, N_{1}, N_{2}\right) \equiv \operatorname{Card}\left\{j \in\left[N_{1}, N_{2}\right]: a_{j} \in I\right\} .
$$

The following is a result regarding equidistributed sequences $[15,16]$ :

Lemma (5.11). For every $M>1$ there exists $N_{0}>1$ such that, if $N \geqslant N_{0}$, then

$$
|| I\left|-\frac{N(I, j, j+N)}{N}\right|<\frac{1}{M}
$$

for all $j \leqslant M N, I=(c, d) \subset[0,1],|I|=|d-c|$. Moreover, for the best equidistributed sequences,

$$
N(M, \mathbf{a}) \leqslant G_{0} M^{-1},
$$

where $N(M, \mathbf{a})$ denotes the infimum of all such $N_{0}$ for a given a. 
For $l \in \mathscr{M}, t \in\left[t_{l}^{0}, t_{l}^{1}\right)$, define

$$
E_{l}(t)=x_{l}(t)-x_{l}\left(t_{l}^{0}\right)-\int_{t_{l}^{0}}^{t} \lambda_{l}[t] d t .
$$

THEOREM (5.12). For each equidistributed sequence a there exists a positive function $f(\mu) \leqslant 1, f(\mu) \rightarrow 0$ as $\mathscr{M} \rightarrow 0$, such that the following two statements hold:

If $h \leqslant f(\mu) \nu, t \in\left[t_{l}^{0}, t_{l}^{1}\right)$, then

$$
\left|\gamma_{l}\right| \leqslant \mu
$$

for all $l \in \mathscr{M}^{+}(J)$ such that $t_{l}^{0}=0, t_{l}^{1} \geqslant \nu$. If $h \leqslant \mathcal{f}(\mu) t_{J}, t \in\left[t_{l}^{0}, t_{l}^{1}\right)$, then

$$
\left|E_{l}(t)\right| \leqslant \mu t_{J}
$$

for all $l \in \mathscr{M}$.

(Here (5.59) says that the strength of rarefaction characteristics tends to zero uniformly with $h$ due to the splitting of characteristics; and (5.60) says that as $h \rightarrow 0$, characteristics move with characteristic speed.)

Proof. First, for $l \in \mathscr{M}$, define (cf. (5.51), Lemma (5.10))

$$
A_{l}(j 1, j 2)=\sum_{j=j 1}^{j 2} G_{0}\left\{B_{l}(j)+B_{l}^{-}(j)+C_{l(j), j}+D_{l(j), j}\right\} .
$$

Set $A \equiv \operatorname{Max}\left\{2, G_{0} V_{0}\right\}$, so that

$$
A_{l}(j 1, j 2) \leqslant G_{0} V_{0} \leqslant A, \quad A \geqslant 2,
$$

for all $j 1, j 2<J$. Let a and $\mu>0$ be given. Let $M$ be the smallest integer such that

$$
M \geqslant 3 G_{0} A(2+k / h) \mu^{-1},
$$

and define

$$
f(\mu)=1 / M N(M, \mathbf{a}),
$$

where in addition to all previous estimates, $G_{0}$ satisfies

$$
\left|\gamma_{l}\right| \leqslant G_{0}\left|\lambda_{p}\left(u_{p}^{L}[t]\right)-\lambda_{p}\left(u_{l}^{R}[t]\right)\right|
$$

for all $l \in \mathscr{M}_{p}^{+}, p=1, \ldots, n, t_{l}^{0} \leqslant t<t_{l}^{1}<t_{J}$. (Recall that the strength $\left|\gamma_{l}(t)\right|$ is constant and equal to $\left|\gamma_{l}\right|$ for all $t_{l}^{0} \leqslant t<t_{l}^{1}$.) Such a $G_{0}$ exists by Property (4.3) together with the assumption of genuine nonlinearity. Here $N(M, a)$ is defined in Lemma (5.11). We first verify (5.59). For this case choose $\nu>0$, and let $l \in \mathscr{M}_{p}^{+}$ satisfy $t_{l}^{1}>\nu$. Fix $h \leqslant f(\mu) \nu$, and set $N=\nu M^{-1} h^{-1}$, so that $h=\nu / M N$, and by definition of $f, N \geqslant N(M$, a). By (5.51),

$$
\sum_{j^{\prime}=0}^{j}\left|\lambda_{l}\left[t_{j^{\prime}}+\right]-\lambda_{l}\left[t_{j^{\prime}}-\right]\right| \leqslant A_{l}(1, j) .
$$

Thus for some integer $m, 0 \leqslant m \leqslant M-1$, we must have

$$
A_{l}(m N,(m+1) N) \leqslant \frac{A_{l}\left(0, j_{l}^{1}\right)}{M} \leqslant \frac{A}{M} .
$$


But this implies that for some $j \in[m N,(m+1) N]$,

$$
x_{i}+a_{j} h \in\left(x_{i}+\lambda_{L}(j) k, x_{i}+\lambda_{L}(j) k+3 A / M\right),
$$

or equivalently,

$$
a_{j} \in\left[\lambda_{l}\left[t_{j}\right], \lambda_{l}\left[t_{j}\right]+3 A / M\right] k / h .
$$

where $i=l(j)$. This follows because (5.66) and (5.67) imply that there is a fixed and open interval $I, I \subset\left[\lambda_{L}(j), \lambda_{L}(j)+3 A / M\right] k / h$ for all $j \in[m N,(m+1) N]$, such that $|I| \geqslant A / M>1 / M$, where we use (5.62). Thus by Lemma (5.11),

$$
N(I, m N,(m+1) N) \geqslant\{|I|-A / M\} N>0,
$$

and so $N(I, m N,(m+1) N) \geqslant 1$. By definition of approximate characteristics, this implies that

$$
\left|\lambda_{p}\left(u_{l}^{R}\left[t_{j}+\right]\right)-\lambda_{p}\left(u_{l}^{L}\left[t_{j}-\right]\right)\right| \leqslant 3 A / M
$$

for that value of $j \in[m N,(m+1) N]$ for which $a_{j} \in I$. Thus by (5.65),

$$
\left|\gamma_{l}\left(t_{j}+\right)\right| \leqslant 3 G_{0} A / M \leqslant \nu .
$$

This verifies (5.59) since $t_{j} \leqslant \nu<t_{l}^{1}$ implies $\left|\gamma_{l}\right|=\left|\gamma_{l}\left(t_{j}+\right)\right|$.

We now verify (5.60). Fix $h \leqslant f(\mu) t_{J}$, and set $N \equiv t_{J} M^{-1} h^{-1}$ so that $h=t_{J} / M N$, and by (5.64) $N \geqslant N(M, \mathrm{a})$.

Without loss of generality, we do the case $l \in \mathscr{M}\left(t_{J}\right) \backslash \mathscr{N}\left(t_{J}\right)$, the case $l \in \mathscr{N}\left(t_{J}\right)$ being similar. Thus let $l \in \mathscr{M}(J) \backslash \mathscr{N}\left(t_{j}\right)$ be fixed. Define

$$
E_{l}(j 1, j 2) \equiv x_{l}\left(t_{j 2}\right)-x_{l}\left(t_{j 1}\right)-\int_{t_{j 1}}^{t_{j 2}} \lambda_{l}[t] d t,
$$

and let $\lambda_{j} \equiv \lambda_{l}\left[t_{j}-\right]$. By the definition of approximate characteristics,

$$
E_{l}(j 1, j 2)=\sum_{j=j 1}^{j 2} E_{l}(j),
$$

where

$$
E_{l}(j)= \begin{cases}\left(\frac{k}{h} \lambda_{j}\right) h, & \text { if } a_{j} \leqslant \frac{k}{h} \lambda_{j}, \\ \left(\frac{k}{h} \lambda_{j}-1\right) h, & \text { if } a_{j}>\frac{k}{h} \lambda_{j} .\end{cases}
$$

For $j \in[1, N],\left|\lambda_{j}-\lambda_{1}\right| \leqslant A_{l}(1, N) \equiv A_{0}$. Define

$$
\begin{aligned}
& \bar{N}_{1}=\left\{j \leqslant N: a_{j} \leqslant \frac{k}{h} \lambda_{j}\right\}, \quad \bar{N}_{2}=\left\{j \leqslant N: a_{j}>\frac{k}{h} \lambda_{j}\right\}, \\
& I_{1}=\left[0,1-\frac{k}{h} \lambda_{1}-A_{0}\right], \quad I_{2}=\left[1-\frac{k}{h} \lambda_{1}+A_{0}, 1\right], \\
& I_{3}=\left[1-\frac{k}{h} \lambda_{1}-A_{0}, 1-\frac{k}{h} \lambda_{1}+A_{0}\right],
\end{aligned}
$$

and set

$$
N_{n} \equiv N\left(I_{n}, 1, N\right), \quad n=1,2,3
$$


Then by (5.70),

Now we can estimate

$$
\bar{N}_{1} \leqslant N_{1}+N_{3}, \quad \bar{N}_{2} \leqslant N_{2}+N_{3} .
$$

$$
\begin{aligned}
\left|E_{l}(1, N)\right| & =\left|\sum_{j=1}^{N} E_{l}(j)\right| \leqslant\left(\frac{k}{h} \lambda_{1}\right) h \bar{N}_{1}-\left(1-\frac{k}{h} \lambda_{1}\right) h \bar{N}_{2}+\frac{k}{h} A_{0} h N \\
& \leqslant\left(\frac{k}{h} \lambda_{1}\right) h N_{1}-\left(1-\frac{k}{h} \lambda_{1}\right) h N_{2}+h N_{3}+\frac{k}{h} A_{0} h N .
\end{aligned}
$$

But since $N=N(\mu, \mathbf{a})$,

$$
N_{1} \leqslant\left(1-\frac{k}{h} \lambda_{1}\right) N+\frac{1}{M} N, \quad N_{2} \geqslant\left(\frac{k}{h} \lambda_{1}\right) N-\frac{1}{M} N, \quad N_{3} \leqslant 2 A_{0} \frac{k}{h} N+\frac{1}{M} N .
$$

Substituting into (5.71) gives

$$
\begin{aligned}
\left|E_{l}(1, N)\right| \leqslant & \left(\frac{k}{h} \lambda_{1}\right) h\left(1-\frac{k}{h} \lambda_{1}\right) N+\left(\frac{k}{h} \lambda_{1}\right) \frac{1}{M} h N \\
& -\left(1-\frac{k}{h} \lambda_{1}\right) h\left(\frac{k}{h} \lambda_{1}\right) N+\left(1-\frac{k}{h} \lambda_{1}\right) \frac{1}{M} h N \\
& +2 \frac{k}{h} A_{0} h N+\frac{1}{M} h N+\frac{k}{h} A_{0} h N \\
\leqslant & 2 \frac{1}{M} h N+3 \frac{k}{h} A_{0} h N .
\end{aligned}
$$

Now since $h N=t_{J} / M$, conclude that

$$
\left|E_{l}(1, N)\right| \leqslant t_{J} \frac{1}{M}\left\{2 \frac{1}{M}+3 \frac{k}{h} A_{l}(0, N)\right\} .
$$

Similarly,

$$
\left|E_{l}(m N+1,(m+1) N)\right| \leqslant t_{J} \frac{1}{M}\left\{2 \frac{1}{M}+3 \frac{k}{h} A_{l}(m N+1,(m+1) N)\right\} .
$$

Therefore,

$$
\begin{aligned}
\left|E_{l}\left(j_{l}^{0}, j_{l}^{1}\right)\right| & \leqslant \sum_{m=1}^{M}\left\{2 \frac{1}{M}+3 \frac{k}{h} A_{l}(m N,(m+1) N)\right\} t_{J} \frac{1}{M} \\
& =\left\{2+3 \frac{k}{h} A_{l}\left(j_{l}^{0}, j_{l}^{1}\right)\right\} t_{J} \frac{1}{M} \\
& \leqslant\left\{2+3 \frac{k}{h} A\right\} t_{J} \frac{1}{M} \leqslant \mu t_{J},
\end{aligned}
$$

thus verifying (5.60). For the best equidistributed sequences, $N(M, \mathbf{a})=G_{0} \mu^{-1}$ by Lemma (5.11), and so in this case $f(\mu)=G_{0} \mu^{2}$ by (5.63) and (5.64). This completes the proof of Theorem (5.12).

6. The main estimate. In this section we study approximate solutions $u^{h}(x, t)$ generated by the random choice method from initial data $u_{0}^{h}(x)$ which satisfies

$$
u_{0}^{h}( \pm \infty)=0 \text {. }
$$

We study the approximate solutions in a coordinate system of Riemann invariants if one exists, and if not, then in a coordinate system that is a good approximation to a coordinate system of Riemann invariants near $u=0$. 
Thus, let $z \equiv\left(z_{1}, \ldots, z_{n}\right)$ denote a coordinate system of Riemann invariants if one exists; i.e., in this case assume that the mapping $u \rightarrow z$ is a 1-1 smooth map taking $0 \rightarrow 0$, and which satisfies the condition

$$
\partial / \partial z_{k} \propto R_{k} .
$$

Such a coordinate system exists if and only if there is a choice of eigenvector fields $\left\{R_{k}^{\prime}\right\}_{k=1}^{n}$ such that $\left[R_{j}^{\prime}, R_{k}^{\prime}\right]=0$ for all $j, k \in\{1, \ldots, n\}$, where [ ] denotes the Lie bracket. A coordinate system of Riemann invariants always exists in the case $n=2$.

If a coordinate system of Riemann invariants does not exist, then choose $z=$ $\left(z_{1}, \ldots, z_{n}\right)$ to satisfy $(\mathrm{cf} .[6,17])$

$$
\left.\frac{\partial}{\partial z_{k}}\right|_{z=0}=R_{k}(0) .
$$

In either case, let $z_{p}$ be the wave strength parametrization of the $p$-shock-rarefaction curve $\mathscr{Y}_{p}\left(u_{L}\right)$ in a neighborhood of $u=0$ (cf. (2.1)).

For example, we take $\sigma \equiv z_{p}(u)-z_{p}\left(u_{L}\right)$ in the equation $u=T_{p}\left(\sigma ; u_{L}\right)$, so that

$$
\gamma^{p} \equiv z_{p}\left(u^{R}\right)-z_{p}\left(u^{L}\right)
$$

defines the signed strength of a $p$-wave $\gamma^{p}$ with left and right states $u^{L}$ and $u^{R}$.

We let $U$ denote a neighborhood of $u=0$ in which Riemann problems are uniquely solvable such that $z_{p}$ is a regular wave strength parameter for all $p$-wave curves in $U$, and such that Lemma (GL1) (cf. (3.15), (3.16)) applies with this measure of wave strength. We say that $V$ is sufficient for $U$ in Lemma (GL2) if any approximate solution $u^{h}$ satisfying (6.1) together with

$$
V_{0} \equiv \sum_{i, p}\left|\gamma_{i 0}^{p}\right|<V
$$

takes values in $U$ for all $x$ and $t$, and satisfies the conclusions (3.18)-(3.20) of Lemma (GL2) as well. We say that $\tilde{M}$ is sufficient for $U$ and $V$ in Lemma (GL3) if

$$
V_{0}<V \text {, }
$$

together with

$$
\left\|u_{0}^{h}(\cdot)\right\|_{s} \leqslant 1 / \tilde{M}
$$

guarantee that $u^{h}$ takes values in $U$ for all $x, t$, and satisfies the conclusions (3.18)-(3.23) of Lemma (GL3) as well. It is clear from the statements of Lemmas (GL2) and (GL3) that for every neighborhood $U$ of $u=0$ there is a $V^{\prime}$ such that any $V<V^{\prime}$ will be sufficient for $U$ in Lemma (GL2); and in the case of a coordinate system of Riemann invariants, for every $U$ and $V$ there is an $\tilde{M}^{\prime}$ such that $\tilde{M}>\tilde{M}^{\prime}$ will be sufficient for $U$ and $V$ in Lemma (GL3).

For $u \in U$, let $|u| \equiv \operatorname{Sup}_{p}\left|z_{p}(u)\right|$, and for functions $u: R \rightarrow U$, define

$$
\begin{gathered}
|u(x)| \equiv \operatorname{Sup}_{p}\left|z_{p}(u(x))\right|, \\
\|u(\cdot)\|_{L^{1}} \equiv \int_{-\infty}^{\infty}|u(x)| d x, \\
\|u(\cdot)\|_{S} \equiv \operatorname{Sup}_{x}\{|u(x)|\} .
\end{gathered}
$$


The following two lemmas will be needed. The first lemma estimates the change in $z_{p^{\prime}}$ across a $p$-wave, $p^{\prime} \neq p$ :

LEMMA (6.1). Let $\gamma^{p}$ be any p-wave with left and right states $u^{L}$ and $u^{R}$ satisfying

$$
\left|u^{q}\right| \leqslant 1 / M, \quad q=L, R .
$$

Then

$$
\left|z_{p^{\prime}}\left(u^{R}\right)-z_{p^{\prime}}\left(u^{L}\right)\right| \leqslant \frac{G_{0}}{M}\left|\gamma^{p}\right| .
$$

Moreover, if $z$ is a coordinate system of Riemann invariants, then

$$
\left|z_{p^{\prime}}\left(u^{R}\right)-Z_{p^{\prime}}\left(u^{L}\right)\right| \leqslant \frac{G_{0}}{M^{2}}\left|\gamma^{p}\right| .
$$

Proof. Statements (6.12) and (6.13) follow directly from (6.3) together with the fact that $\mathscr{S}_{p}\left(u^{L}\right)$ makes $C^{2}$ P.W. $C^{3}$ contact with $\mathscr{R}_{p}\left(u^{L}\right)$ at the state $u^{L}$ (cf. (2.1)).

It is clear from (13) that if $U$ is sufficiently small, then

$$
\left|z_{p}\left(u^{R}\right)-z_{p}\left(u^{L}\right)\right|>2\left|z_{p^{\prime}}\left(u^{R}\right)-z_{p^{\prime}}\left(u^{L}\right)\right|
$$

for all $p$-waves with left and right states $u^{L}$ and $u^{R}$ in $U, p^{\prime} \neq p$.

The next lemma is a technical but elementary uniform estimate for the speed of a $p$-shock in terms of $z_{p}$.

LEMMA (6.2). Let $S$ denote a p-shock with speed $s$ and left and right states $u^{L}$ and $u^{R}$. Then there exist a constant $M_{1}>0$ and a constant $G_{0}>0$ depending only on $M_{1}$ and $f$, such that, if $M>M_{1}$ and

$$
\begin{gathered}
\left|u^{q}\right|<\frac{1}{M}, \quad q=L, R, \\
z_{p}(\bar{u})-\frac{z_{p}\left(u^{L}\right)+z_{p}\left(u^{R}\right)}{2} \geqslant \frac{1}{16 M},
\end{gathered}
$$

and

$$
\left|z_{p^{\prime}}(\bar{u})-z_{p^{\prime}}\left(u^{R}\right)\right| \leqslant \frac{1}{G_{0} M}
$$

for all $p^{\prime} \neq p$, then

$$
\lambda_{p}(\bar{u})-s \geqslant \frac{1}{G_{0} M}
$$

Proof. Lemma (6.2) expresses in a uniform way the fact of Lemma (2.1) that

$$
s=\lambda_{p}\left(\frac{u^{L}+u^{R}}{2}\right)+0(1)|S|^{2} .
$$

The existence of $M_{1}$ follows from (6.3) and the uniformity follows from the compactness of $U$ together with the fact that $\left(\partial / \partial z_{p}\right) \lambda_{p}>0$.

In the analysis to follow, it is important to bound changes in $\lambda_{p}$ by changes in $z_{p}$. This can be done because $z \rightarrow u$ is a regular map, and $\nabla \lambda_{p} \cdot R_{p}>0$. Define

$$
\lambda \equiv \operatorname{Inf}_{\sigma, u^{L}} \frac{\partial \lambda_{p}\left(\mathscr{T}_{p}\left(\sigma ; u^{L}\right)\right)}{\partial \sigma}
$$


where $\sigma=z_{p}(u)-z_{p}\left(u^{L}\right)$ and the infimum is taken over all values of $\sigma, u^{L}$ such that $u=\mathscr{T}_{p}\left(\sigma ; u^{L}\right) \in U$.

Now let $G_{0}$ be large enough to satisfy all previous conditions, as well as

$$
G_{0} \geqslant \operatorname{Max}\left\{1, \lambda^{-1}, V\right\} \text {. }
$$

Define the following constants:

$$
\begin{aligned}
\delta & \equiv \delta\left(G_{0}\right)=1 / 64 G_{0}^{2} . \\
G & \equiv G\left(\delta, G_{0}\right)=1 / \delta^{2}, \\
M_{2} & \equiv M_{2}\left(\delta, G_{0}\right)=G_{0}^{2} / \delta .
\end{aligned}
$$

Choose $U$ to be a sufficiently small neighborhood of $u=0$ so that (6.14) holds and

$$
U \subseteq\left\{u:|u| \leqslant M_{i}^{-1}\right\}
$$

for $i=1,2$. We prove the following theorem, which is a restatement of Lemmas (2A), (2B) of the Introduction.

THEOREM (6.3). Let $u^{h}$ be a fixed random choice method approximate solution satisfying (1). Define

$$
\begin{gathered}
1 / M \equiv\left\|u_{0}^{h}(\cdot)\right\|_{S}, \\
\varepsilon \equiv\left\|u_{0}^{h}(\cdot)\right\|_{L^{1}}, \\
T \equiv \varepsilon(G M)^{2} .
\end{gathered}
$$

In the case that $z$ is not a coordinate system of Riemann invariants, assume that

$$
V_{0} \leqslant V \text {, }
$$

where $V$ is sufficient for $U$ in Lemma (GL2) and

$$
V \leqslant \delta / G_{0}^{2} \text {. }
$$

In the case that $z$ is a coordinate system of Riemann invariants, assume that $V$ is arbitrary and that

$$
V_{0} \leqslant V
$$

and

$$
M \geqslant \tilde{M},
$$

where $\tilde{M}$ is sufficient for $U$ and $V$ in Lemma (GL3). Under these assumptions, if

$$
h<\operatorname{Min}\left\{\frac{T \delta^{2}}{G_{0} M^{2}},\left(\frac{T^{1 / 2} \lambda}{30 \sqrt{2} G_{0}^{1 / 2}}\right),\left(\frac{T^{1 / 2}}{(12 \sqrt{2} M) G_{0}^{3 / 2}}\right)^{2}\right\},
$$

then

$$
Q(0)-Q(T) \geqslant 1 /(G M)^{2},
$$

where $Q$ is the quadratic Glimm functional associated with $u^{h}(c f .(3.11))$. Note that by (6.25), any $u^{h}$ that satisfies either (6.29) or (6.31), (6.32) must also satisfy $\left\|u^{h}(\cdot, t)\right\|_{S}$ $\leqslant 1 / M_{2}$ for $i=1,2$, so in particular

$$
M \geqslant G_{0}^{2} / \delta .
$$


The remainder of this section is devoted to the proof of Theorem (6.3). From here on assume that $u^{h}$ is a given random choice method approximate solution that satisfies (6.1), (6.33) and either (6.29), (6.30) or (6.30), (6.31). Let $\Gamma$ and $\mathscr{M}$ denote the characteristics and index set associated with $u^{h}$ and the time level $t_{J}$, where

$$
J \equiv \operatorname{Min}\{[T / k]+1,[2 T / k]\} \text {. }
$$

(Here [ ] denotes "greatest integer in".)

The choice of $z_{p}$ for the $p$-wave strength parameter determines the definition of wave strength for the characteristics in $\Gamma$ (cf. (4.6)). In this case, for $l \in \mathscr{M}_{p}$,

$$
\gamma_{l}(t) \equiv z_{p}\left(u_{l}^{R}[t]\right)-z_{p}\left(u_{l}^{L}[t]\right)
$$

defines the signed strength of the characteristic $\gamma_{l}$ at time $t<t_{J}$. Recall that $\gamma_{l}(t) \equiv \gamma_{l}$ is constant for $t \in\left[t_{l}^{0}, t_{l}^{1}\right)$, and identically zero elsewhere.

The idea in the proof to follow is this: Since $\left\|u_{0}(\cdot)\right\|_{S}=1 / M$ and $\left\|u_{0}(\cdot)\right\|_{0}=\varepsilon$, there must be a "spike" in the initial data of height $1 / M$ and width on the order of $\varepsilon M$. We use this to construct two characteristics $\gamma_{r} \in \Gamma_{p}^{+}$and $\gamma_{s} \in \Gamma_{p}^{-}$, which would intersect before time $T$ if there were no interactions to deflect the speeds of these characteristics from their initial speeds at time $t=0$. We then assume that $Q(0)-$ $Q(T) \leqslant 1 /(G M)^{2}$. By this assumption, there exists such $\gamma_{r}, \gamma_{s}$ which are not cancelled in time $[0, T]$. We then use the same assumption to obtain estimates for the change in the speeds of $\gamma_{r}$ and $\gamma_{s}$ between time $t=0$ and $t=T$. These estimates are sufficient to guarantee that in time $[0, T]$ the wave speeds of $\gamma_{r}$ and $\gamma_{s}$ are not deflected enough from their initial speeds to prevent them from intersecting before time $T$. This intersection implies that one of them is cancelled before time $T$ (cf. Property (4.1)). This is a contradiction and thus we conclude that $Q(0)-Q(T) \geqslant$ $1 /(G M)^{2}$. We first use Lemma (6.1) together with (6.30) or (6.24) to estimate the total variation in $z_{p}$ contributed by characteristics not in the $p$-family.

LEMMA (6.4). Our assumptions on $u^{h}$ imply

$$
\sum_{\mathscr{M}_{\mathscr{M}_{p}}}\left|z_{p}\left(u_{l}^{R}[0]\right)-z_{p}\left(u_{l}^{L}[0]\right)\right| \leqslant \delta / M
$$

Proof. A restatement of Lemma (6.1) in the language of approximate characteristics is that

$$
\left|z_{p}\left(u_{l}^{R}[0]\right)-z_{p}\left(u_{l}^{L}[0]\right)\right| \leqslant G_{0}\left|\gamma_{l}(0)\right| / M
$$

for all $l \in \mathscr{M} \backslash \mathscr{M}_{p}$; and if $z$ is a coordinate system of Riemann invariants, then

$$
\left|z_{p}\left(u_{l}^{R}[0]\right)-z_{p}\left(u_{l}^{L}[0]\right)\right| \leqslant G_{0}\left|\gamma_{l}(0)\right| / M^{2} \text {. }
$$

But by Property (4.2)

$$
\sum_{\mathscr{M}}\left|\gamma_{l}(0)\right|=V_{0} \leqslant V
$$

Thus statement (6.36) follows directly from either (6.37A) or (6.37B) by estimating the right-hand sides using either (6.30) or (6.34A), respectively.

By (6.26) there exists $p, 1 \leqslant p \leqslant n$, such that

$$
1 / M=\operatorname{Sup}_{x}\left|z_{p}\left(u_{0}^{h}(x)\right)\right| \text {. }
$$


For convenience we assume that

$$
1 / M=\operatorname{Sup}_{x}\left\{z_{p}\left(u_{0}^{h}(x)\right)\right\}
$$

the case when $1 / M=-\operatorname{Inf}_{x}\left\{z_{p}\left(u_{0}^{h}(x)\right)\right\}$ being handled analogously.

By (6.39) there exists a point $\bar{x}=\left(\bar{i}+\frac{1}{2}\right) h$ such that

$$
z_{p}\left(u_{0}^{h}(\bar{x})\right) \geqslant(1-\delta) / M
$$

Define $i_{A}$ and $i_{B}$ by

$$
\begin{aligned}
& i_{A} \equiv \operatorname{Sup}\left\{i: i<\bar{i}+\frac{1}{2} \text { and } z_{p}\left(u_{0}^{h}\left(x_{i}-\right)\right) \leqslant \delta / M\right\}, \\
& i_{B} \equiv \operatorname{Inf}\left\{i: i>\bar{i}+\frac{1}{2} \text { and } z_{p}\left(u_{0}^{h}\left(x_{i}+\right)\right) \leqslant \delta / M\right\} .
\end{aligned}
$$

Let

$$
x_{A} \equiv\left(i_{A}-\frac{1}{2}\right) h, \quad x_{B}=\left(i_{B}+\frac{1}{2}\right) h .
$$

Define $\mathscr{R} \subseteq \mathscr{M}_{p}^{-}$and $\mathscr{S} \subseteq \mathscr{M}_{p}^{+}$by

$$
\begin{aligned}
& \mathscr{R} \equiv\left\{l \in \mathscr{M}_{p}^{+}: x_{l}(0) \in\left(x_{1}, \bar{x}\right)\right\}, \\
& \mathscr{S} \equiv\left\{l \in \mathscr{M}_{p}^{-}: x_{l}(0) \in\left(\bar{x}, x_{2}\right)\right\},
\end{aligned}
$$

and let (cf. (5.1))

$$
\mathscr{R}_{0} \equiv \mathscr{R} \backslash \mathscr{N}(T), \quad \mathscr{S}_{0} \equiv \mathscr{S} \mathscr{N}(T)
$$

LEMMA (6.5). The following estimate holds:

$$
\left|x_{B}-x_{A}\right| \leqslant \varepsilon M / \delta .
$$

Proof. By (6.40),

$$
\varepsilon=\int_{-\infty}^{\infty}\left|u_{0}^{h}(x)\right| d x \geqslant \delta\left|x_{B}-x_{A}\right| / M .
$$

Solving for $\left|x_{B}-x_{A}\right|$ gives (6.45).

Proposition (6.6). If $Q(0)-Q(T) \leqslant 1 /(G M)^{2}$, then there exist $r \in \mathscr{R}_{0}$ and $s \in \mathscr{S}_{0}, s_{0}=i_{s}$, such that the following conditions hold:

$$
\left\{z_{p}\left(u_{r}^{L}[0]\right), z_{p}\left(u_{r}^{R}[0]\right)\right\} \subseteq(3 / 4 M, 7 / 8 M),
$$

and either

$$
z_{p}\left(u_{s}^{R}[0]\right) \leqslant 3 / 8 M
$$

and

$$
\left|\gamma_{i_{s} 0}^{p}\right| \geqslant \delta / M
$$

or else

$$
z_{p}\left(u_{s}^{R}[0]\right) \in(1 / 8 M, 1 / 4 M)
$$

and there exist $i_{L}, i_{R}$ with $i_{A} \leqslant i_{L}<i_{s}<i_{R} \leqslant i_{B}$ such that

$$
\left|\gamma_{i 0}^{p}\right| \leqslant \delta / M
$$


for all $i \in \mathscr{M}_{p}^{-}\left[i_{L}, i_{R}\right]$, and $(c f .(5.41))$

$$
\begin{gathered}
\sum_{\mathscr{M}_{p}^{-}\left[i_{L}, i_{s}\right]}\left|\gamma_{i}\right| \geqslant 4 \delta / M, \\
\sum_{\mathscr{M}_{p}^{-}\left[i_{s}, i_{R}\right]}\left|\gamma_{i}\right| \geqslant 4 \delta / M .
\end{gathered}
$$

Proof. We first verify the existence of $r \in \mathscr{R}_{0}$ satisfying (6.46). Let

$$
\mathscr{L} \equiv\left\{l \in \mathscr{M}: x_{l}(0) \in\left[x_{A}, \bar{x}\right]\right\},
$$

and let $I_{l}$ denote the interval $\left[z_{p}\left(u_{l}^{L}[0]\right), z_{p}\left(u_{l}^{R}[0]\right)\right]$. Let $\left|I_{l}\right|$ denote the length of the interval $I_{l}$. Let

$$
m \equiv \operatorname{Inf}\left\{z\left(u_{0}^{h}(x)\right): x \in\left[x_{A}, \bar{x}\right]\right\}
$$

By (6.39) and (6.41),

$$
-M \leqslant m \leqslant \delta / M .
$$

Property (4.3) states that the approximate characteristics partition the waves in the approximate solution $u^{h}$ at each time step. This together with (6.40) implies

$$
\bigcup_{\mathscr{L}} I_{l} \supseteq[m,(1-\delta) / M] \text {. }
$$

By Lemma (6.4),

$$
\sum_{\mathscr{L} \backslash \mathscr{M}_{p}}\left|I_{l}\right|<\delta / M
$$

and since we assume $Q(0)-Q(T) \leqslant 1 /(G M)^{2}$, Corollary (5.8) implies

$$
\sum_{\mathscr{L} \cap \mathcal{N}(T)}\left|I_{l}\right|<\delta / M
$$

Since $z_{p}\left(u_{l}^{R}[0]\right)-z_{p}\left(u_{l}^{L}[0]\right)$ is positive for $l \in \mathscr{M}^{+}$and negative for $l \in \mathscr{M}^{-}$, and since $z_{p}$ increases from $m$ to $(1-\delta) / M$ going from $x_{A}$ to $\bar{x}$ at time $t=0$, we can write

$$
\bigcup_{\mathscr{L}} I_{l}=\left\{\bigcup_{\mathscr{R}} I_{l}\right\} \cup\left\{\bigcup_{\mathscr{L} \backslash \mathscr{M}_{p}} I_{l}\right\} .
$$

Since $\mathscr{R} \subseteq \mathscr{R}_{0} \cup\{\mathscr{L} \cap \mathscr{N}(T)\},(6.57),(6.58)$ and (6.59) imply

$$
\bigcup_{\mathscr{R}_{0}} I_{l} \supset[0,1 / M] \backslash X
$$

where $X$ is a set of small measure,

$$
m\{X\}<4 \delta / M<1 / 16 M,
$$

where we have used (6.22) to estimate $\delta$.

By (5.59), if $r \in \mathscr{R}_{0}$, then

$$
\left|\gamma_{r}\right|<1 / 16 M
$$

because $h<G_{0}^{-1}(1 / 16 M)^{2} T$ by (6.14). Thus (6.60)-(6.62) immediately imply the existence of $r \in \mathscr{R}_{0}$ such that (6.46) holds. 
Similarly, we conclude as in (6.60) that

$$
\bigcup_{\mathscr{S}_{0}} I_{l} \supseteq[0,1 / M] \backslash X,
$$

where $X$ satisfies (6.61), which directly implies the existence of $s \in \mathscr{S}_{0}$ satisfying either (6.47), (6.48) or (6.49), (6.50).

Define (cf. (5.38)-(5.40))

$$
\begin{gathered}
\mathscr{R}_{0}^{L} \equiv\left\{l \in \mathscr{R}_{0}: x_{l}(0) \leqslant x_{r}(0)\right\} \\
\mathscr{S}_{0}^{R} \equiv\left\{l \in \mathscr{S}_{0}: x_{l}(0) \geqslant x_{s}(0)\right\}, \\
\mathscr{B}_{0} \equiv\left\{l \in \mathscr{M}_{p^{\prime}}: p^{\prime} \neq p \text { and } \gamma_{l} \text { intersects } \gamma_{s} \text { in }[0, T / 2]\right\}, \\
B_{0}=\sum_{\mathscr{B}_{0}}\left|\gamma_{l}\right|, \\
\mathscr{B}_{r} \equiv \mathscr{B}_{r}[0, T / 2], \quad B_{r} \equiv \sum_{\mathscr{B}_{r}}\left|\gamma_{l}\right|, \\
\mathscr{B}_{s} \equiv \mathscr{B}_{s}[0, T / 2], \quad B_{s} \equiv \sum_{\mathscr{B}_{r}}\left|\gamma_{l}\right|, \\
\mathscr{B}_{s q}^{-} \equiv \mathscr{B}_{s q}^{-}[0, T / 2], \quad B_{s q}^{-} \equiv \sum_{\mathscr{B}_{s q}^{-}}\left|\gamma_{l}\right|, \quad q=L, R \text { or absent. }
\end{gathered}
$$

Theorem (6.3) is a direct consequence of Proposition (6.6) together with

Proposition (6.7). If $Q(0)-Q(T)<1 /(G M)^{2}$ where $u^{h}$ satisfies our previous assumptions, then either $r \in \mathcal{N}(T)$ or $s \in \mathcal{N}(T)$.

Proof of Theorem (6.3). If $Q(0)-Q(T)<1 /(G M)^{2}$, then by Proposition (6.7) either $r \in \mathscr{N}(T)$ or $s \in \mathcal{N}(T)$. But by Proposition (6.6) both $r$ and $s$ are in $\mathscr{M} \backslash \mathscr{N}(T)$. Thus by contradiction we must have $Q(0)-Q(T) \geqslant 1 /(G M)^{2}$.

It remains to give a proof of Proposition (6.7). Proposition (6.7) is a consequence of the following lemmas. The idea is to show that if $Q(0)-Q(T)<1 /(G M)^{2}$, then for $t<T, \lambda_{r}[t]$ and $\lambda_{s}[t]$ are sufficiently close to $\lambda_{r}[0]$ and $\lambda_{s}[0]$, respectively, to guarantee that the characteristics $\gamma_{r}$ and $\gamma_{s}$ must intersect before time $T$. Then by Proposition (5.2), $r \in \mathscr{N}(T)$ or $s \in \mathscr{N}(T)$.

Lemma (6.8). Assume that $Q(0)-Q(T) \leqslant 1 /(G M)^{2}$. Then

$$
\begin{aligned}
& \sum_{\mathscr{R}_{0}^{L}}\left|\gamma_{l}\right| \geqslant \delta / M, \\
& \sum_{\mathscr{S}_{0}^{R}}\left|\gamma_{l}\right| \geqslant \delta / M .
\end{aligned}
$$

Proof. By Property (4.2),

$$
T V\left\{z_{p}\left(u_{0}^{h}(0)\right)\right\}=\sum_{\mathscr{M}}\left|z_{p}\left(u_{l}^{R}[0]\right)-z_{p}\left(u_{l}^{L}[0]\right)\right| .
$$

This together with (6.40)-(6.43) and (6.46) implies that

$$
\sum_{\mathscr{R}^{L}}\left|\gamma_{l}\right| \geqslant 3 / 4 M-\delta / M \text {. }
$$


(Here we use the fact that $z_{p}\left(u_{l}^{R}[t]\right)-z_{p}\left(u_{l}^{L}[t]\right)$ is positive [negative] for $l \in \mathscr{M}_{p}^{+}$ $\left[l \in \mathscr{M}_{p}^{-}\right.$, respectively]). But

$$
\sum_{\mathscr{R}^{L}}\left|\gamma_{l}\right| \geqslant \sum_{\mathscr{R}_{0}^{L}}\left|\gamma_{l}\right|-\sum_{\mathscr{S}(T)}\left|\gamma_{l}\right|
$$

and by Corollary (5.8)

$$
\sum_{\mathcal{N}(T)}\left|\gamma_{l}\right| \leqslant G_{0} / G M \leqslant \delta / M
$$

since we assume $Q(0)-Q(T) \leqslant 1 /(G M)^{2}$. Thus by (6.22), $\delta<1 / 32$, so

$$
\sum_{\mathscr{R}_{0}^{L}}\left|\gamma_{l}\right| \geqslant 3 / 4 M-2 \delta / M \geqslant \delta / M \text {. }
$$

Similarly, in the case (6.49), (6.50) of Proposition (6.6), we must have

$$
\sum_{\mathscr{S}^{R}}\left|\gamma_{l}\right|=\sum_{\mathscr{S}^{R}}\left|\gamma_{l}\right|-\sum_{\mathcal{N}(T)}\left|\gamma_{l}\right| \geqslant 1 / 8 M-\delta / M-\delta / M \geqslant \delta / M .
$$

Since $s \in \mathscr{S}_{0}^{R}$, the case (6.47), (6.48) immediately gives the conclusion (6.71). This completes the proof of Lemma (6.8).

Lemma (6.9A). If $l \in \mathscr{M}_{p^{\prime}} \backslash \mathscr{N}(T), p^{\prime} \neq p$, and $x_{l}(0) \in\left(x_{r}(0), x_{s}(0)\right)$, then in time $[0, T], \gamma_{1}$ either intersects all the characteristics in $\mathscr{R}_{0}^{L}\left(\right.$ the case $\left.p^{\prime}<p\right)$ or else it intersects all the characteristics in $\mathscr{S}_{0}^{R}$ (the case $p^{\prime}>p$ ).

LeMma (6.9B). If $l \in \mathscr{M}_{p^{\prime}} \backslash \mathscr{N}(T), p^{\prime} \neq p$, and $\gamma_{l}$ intersects $\gamma_{r}$ or $\gamma_{s}$ in time $[0, T / 2]$, then in time $[0, T], \gamma_{l}$ either intersects all the characteristics in $\mathscr{R}_{0}^{L}$ or else it intersects all the characteristics in $\mathscr{S}_{0}^{R}$.

Proof. Since a is assumed to be equidistributed, statement (5.60) of Theorem (5.12) implies that for any $l \in \mathscr{M}_{p} \backslash \mathscr{N}(T), 1 \leqslant p \leqslant n$, and $t \leqslant T$,

$$
\left|x_{l}(t)-x_{l}(0)-\int_{0}^{t} \lambda_{l}[t] d t\right| \leqslant \mu t_{J}=\left(G_{0} h t_{J}\right)^{1 / 2} .
$$

Thus

$$
\left|x_{l}(t)-x_{l}(0)-\lambda_{p} t\right| \leqslant\left(G_{0} h t_{J}\right)^{1 / 2}+G_{0} T / M \equiv E,
$$

where without loss of generality we have taken $G_{0}$ large enough so that

$$
\left|\lambda_{l}(t)-\lambda_{p}\right| \leqslant G_{0} / M
$$

for all $l \in \mathscr{M}_{p}(J), t<t_{J}$.

For the proof of Lemma (6.9A), assume that $l \in \mathscr{M}_{p^{\prime}} \backslash \mathcal{N}(T), p^{\prime} \neq p$. We do the case $p^{\prime}<p$ and $\gamma_{l}$ intersects $\gamma_{r}$ in time [0,T/2]; i.e., we show that $x_{l}(T)-x_{r^{\prime}}(T)$ $\leqslant 0$ for all $r^{\prime} \in \mathscr{R}_{0}^{L}$ (cf. Proposition (5.1)). By (6.72),

$$
\begin{aligned}
x_{l}(T)-x_{r^{\prime}}(T)= & x_{l}(T)-x_{r}(T)+x_{r}(T)-x_{r^{\prime}}(T) \\
\leqslant & {\left[x_{l}(0)-x_{r}(0)\right]+\left[\lambda_{p^{\prime}}-\lambda_{p}\right] T+2 E } \\
& +\left[x_{r}(0)-x_{r^{\prime}}(0)\right]+\left[\lambda_{p}-\lambda_{p}\right] T+2 E .
\end{aligned}
$$

Since $\gamma_{l}$ intersects $\gamma_{r}$ in time [0,T/2], (6.72) also implies

$$
0 \geqslant x_{l}(T / 2)-x_{r}(T / 2) \geqslant\left[x_{l}(0)-x_{r}(0)\right]+\left[\lambda_{p^{\prime}}(0)-\lambda_{p}(0)\right] T / 2-E .
$$


Moreover, by Lemma (6.5),

$$
\left[x_{r}(0)-x_{r^{\prime}}(0)\right] \leqslant\left|x_{B}-x_{A}\right| \leqslant \varepsilon M / \delta .
$$

Therefore substituting into (6.73) yields

$$
\begin{aligned}
x_{l}(T)-x_{r^{\prime}}(T) & \leqslant\left[\lambda_{p^{\prime}}(0)-\lambda_{p}(0)\right] T / 2+\{\varepsilon M / \delta+5 E\} \\
& \leqslant-\lambda T / 2+\left\{\varepsilon M / \delta+5\left(G_{0} h t_{J}\right)^{1 / 2}+5 G_{0} T / M\right\} \\
& =\left\{-1+2 \varepsilon M / \delta T \lambda+10\left(G_{0} h t_{J}\right)^{1 / 2} / T \lambda+10 G_{0} / M \lambda\right\} T \lambda / 2 .
\end{aligned}
$$

By (6.28) and (6.34A) we have

$$
\frac{2 \varepsilon M}{\delta T \lambda} \leqslant \frac{2}{\delta G^{2} M \lambda} \leqslant \frac{2}{\delta G^{2}\left(G_{0}^{2} / \delta\right) \lambda}<\frac{1}{3}
$$

by (6.33), $h<\left(T^{1 / 2} \lambda / 30 \sqrt{2} G_{0}^{1 / 2}\right)^{2}$, so

$$
\frac{10}{T \lambda}\left(G_{0} h t_{J}\right)^{1 / 2} \leqslant\left(\frac{10 \sqrt{2} G_{0}^{1 / 2}}{T^{1 / 2} \lambda}\right) h^{1 / 2}<\frac{1}{3}
$$

and by (6.34A) again,

$$
10 G_{0} / M \lambda<1 / 3 .
$$

Thus (6.74) implies $x_{l}(T)-x_{r^{\prime}}(T)<0$. This completes the proof of Lemma (6.9A).

For the proof of Lemma (6.9B), assume $l \in \mathscr{M}_{p^{\prime}} \backslash \mathcal{N}(T), p \neq p^{\prime}$. We do the case $p^{\prime}<p$; i.e., we show that if $l \in \mathscr{M}_{p^{\prime}}, p^{\prime}<p$, and $x_{l}(0) \in\left(x_{r}(0), \bar{x}\right)$, then $\gamma_{l}$ intersects $\gamma_{r^{\prime}}$ in time $[0, T]$ for all $r^{\prime} \in \mathscr{R}_{0}^{L}$. It suffices to show that $x_{l}(T)-x_{r^{\prime}}(T)$ $\leqslant 0$.

$$
\begin{aligned}
& x_{l}(T)-x_{r^{\prime}}(T) \leqslant\left[x_{l}(0)-x_{r^{\prime}}(0)\right]+\left[\lambda_{p}^{\prime}-\lambda_{p}\right] T+2 E \\
& \quad \leqslant \frac{\varepsilon M}{\delta}-\lambda T+2 E=\left\{-1+\frac{\varepsilon M}{\delta T \lambda}+\frac{2}{T \lambda}\left(G_{0} h T_{J}\right)^{1 / 2}+\frac{2 G_{0}}{M \lambda}\right\} T \lambda<0,
\end{aligned}
$$

where we have applied (6.75)-(6.77). This completes the proof of Lemma (6.9B).

Lemma (6.10). Assume that $Q(0)-Q(T) \leqslant 1 /(G M)^{2}$. Then for $q=0, r$ and $s$ $(c f .(6.63)-(6.69))$

$$
B_{q} \leqslant \delta / M
$$

Proof. Write

$$
B_{q} \equiv \sum_{\mathscr{B}_{q}}\left|\gamma_{l}\right|=\sum_{\mathscr{B}_{q} \backslash \mathscr{N}(T)}\left|\gamma_{l}\right|+\sum_{\mathscr{B}_{q} \backslash \mathscr{N}(T)}\left|\gamma_{l}\right| .
$$

By Corollary (5.8),

$$
\sum_{\mathscr{B}_{q} \cap \mathcal{N}(T)}\left|\gamma_{l}\right| \leqslant G_{0} / G M
$$

since we assume that $Q(0)-Q(T) \leqslant 1 /(G M)^{2}$. On the other hand, if $l \in$ $\mathscr{B}_{q} \backslash \mathscr{N}(T)$, then Lemma (6.9) implies that $\gamma_{l}$ intersects all the characteristics in either $\mathscr{R}_{0}^{L}$ or $\mathscr{S}_{0}^{R}$ in time $[0, T]$. For example, assume the case $\mathscr{R}_{0}^{L}$, and define

$$
\mathscr{A}^{\prime} \equiv\left\{\left\langle l, r^{\prime}\right\rangle: l \in \mathscr{B}_{q} \backslash \mathscr{N}(T) \text { and } r^{\prime} \in \mathscr{R}_{0}^{L}\right\} .
$$


Then $\mathscr{A}^{\prime} \subseteq \mathscr{A}(0) \backslash \mathscr{A}(T)$. Thus Lemma (5.7) implies

$$
\sum_{\mathscr{A}^{\prime}}\left|\gamma_{l}\right|\left|\gamma_{r^{\prime}}\right| \leqslant G_{0} / G^{2} M^{2}
$$

since we assume $Q(0)-Q(T) \leqslant 1 /(G M)^{2}$. But by Lemma (6.8),

$$
\sum_{\mathscr{A}^{\prime}}\left|\gamma_{l}\right|\left|\gamma_{r^{\prime}}\right|=\frac{1}{2}\left\{\sum_{\mathscr{B}_{q} \backslash \mathcal{N}(T)}\left|\gamma_{l}\right|\right\}\left\{\sum_{\mathscr{R}_{0}^{L}}\left|\gamma_{l}\right|\right\} \geqslant \frac{\delta}{2 M} \sum_{\mathscr{B}_{q} \backslash \mathscr{N}(T)}\left|\gamma_{l}\right| .
$$

Combining (6.80) and (6.81) gives

$$
\sum_{\mathscr{B}_{q} \backslash \mathscr{N}(T)}\left|\gamma_{l}\right| \leqslant 2 G_{0} / \delta G^{2} M
$$

and combining (6.80) and (6.82) gives

$$
B_{q} \leqslant 4 G_{0} / \delta G^{2} M \leqslant \delta / M,
$$

where we have applied (6.22) and (6.23). This completes the proof of Lemma (6.10).

LEMMA (6.11). If $Q(0)-Q(T) \leqslant 1 /(G M)^{2}$, then

$$
B_{s q}^{-} \leqslant \delta / M
$$

for $q=L$ and $R$.

Proof. First assume that (6.48) of Proposition (6.6) holds, and write

$$
B_{s}^{-}=\sum_{\mathscr{B}_{s}^{-}}\left|\gamma_{l}\right|=\sum_{\mathscr{B}_{s}^{-} \cap \mathscr{N}(T)}+\sum_{\mathscr{B}_{s}^{-} \backslash \mathscr{N}(T)}
$$

By Corollary (5.8),

$$
\sum_{\mathscr{B}_{s}^{-} \cap \mathscr{N}(T)}\left|\gamma_{l}\right| \leqslant G_{0} / G M
$$

and moreover (as in the proof of Lemma (6.10)), $\mathscr{A}^{\prime} \subseteq \mathscr{A}(0) \backslash \mathscr{A}(T)$ where

$$
\mathscr{A}^{\prime} \equiv\left\{\left\langle l, s^{\prime}\right\rangle: l \in \mathscr{B}_{s}^{-}, s^{\prime} \in \mathscr{S}_{0}^{R}, x_{s^{\prime}}(0)=x_{s}(0)\right\} .
$$

(Here we apply Lemma (5.2).) Thus as in (6.80)-(6.82), Lemma (5.7) implies that

$$
\mathscr{B}_{s q}^{-} \leqslant \mathscr{B}_{s}^{-} \leqslant 4 G_{0} / \delta G^{2} M \leqslant \delta / M, \quad q=L, R,
$$

where we apply (6.48) in place of Lemma (6.8).

Now assume (6.49)-(6.52) of Proposition (6.6) holds. In this case (6.50)-(6.52) satisfy the hypotheses (5.42), (5.43) of Proposition (5.9) with $L=\delta / M$. Since we assume $Q(0)-Q(T) \leqslant 1 /(G M)^{2}$, and $1 /(G M)^{2}=\delta^{2}(\delta / M)^{2} \leqslant G_{0}(\delta / M)^{2}$, we can conclude $\mathscr{B}_{s q}^{-} \leqslant \delta / M$ for $q=L, R$. This completes the proof of Lemma (6.11).

Lemma (6.12). Assume that $Q(0)-Q(T) \leqslant 1 /(G M)^{2}$. Then

$$
\lambda_{r}[0]-\lambda_{s}[0] \geqslant 1 / G_{0} M \text {. }
$$

Proof. We prove Lemma (6.12) by satisfying the hypotheses of Lemma (6.2). First, (6.46) implies

$$
z_{p}\left(u_{r}^{L}[0]\right) \geqslant 3 / 4 M
$$


and (6.47), (6.49) imply

$$
z_{p}\left(u_{s}^{R}[0]\right) \leqslant 3 / 8 M \text {. }
$$

Let $\bar{u} \equiv u_{r}^{L}[0]$, and let $S$ denote the $p$-shock $S \equiv \gamma_{i_{s} 0}$. Let $\left\{u^{L}, u^{R}\right\}$ denote the left and right states, and $\sigma$ the speed of the $p$-shock $S$. By (5.49), $\lambda_{s}[0]=\sigma$, and (6.86) gives

$$
0 \leqslant z_{p}\left(u^{R}\right) \leqslant z_{p}\left(u_{s}^{R}[0]\right) \leqslant 3 / 8 M .
$$

By (6.39) we must have $z_{p}\left(u^{L}\right) \leqslant 1 / M$. Thus

$$
\frac{z_{p}\left(u^{L}\right)+z_{p}\left(u^{R}\right)}{2} \leqslant \frac{11}{8 M} .
$$

By (6.85)

$$
z_{p}(\bar{u})-\frac{z_{p}\left(u^{L}\right)+z_{p}\left(u^{R}\right)}{2} \geqslant \frac{1}{16 M} .
$$

Moreover, for $p^{\prime} \neq p$, the difference in $z_{p^{\prime}}$ between $u^{R}$ and $\bar{u}$ is bounded by the total variation in $z_{p^{\prime}}$ of all waves that lie between $x_{r}(0)$ and $x_{s}(0)$ at $t=0$. By Property (4.3), this can be estimated by

$$
\begin{aligned}
\left|z_{p^{\prime}}(\bar{u})-z_{p^{\prime}}\left(u^{R}\right)\right| & \leqslant B_{0}+\sum_{\mathscr{M}_{p}}\left|z_{p^{\prime}}\left(u_{l}^{R}[0]\right)-z_{p^{\prime}}^{L}\left(u_{l}^{L}[0]\right)\right| \\
& \leqslant \frac{\delta}{M}+\frac{\delta}{M}=\frac{2 \delta}{M}
\end{aligned}
$$

where we have applied Lemmas (6.4) and (6.10) in the second inequality. Thus Lemma (6.2) applies with $L=1 / M$ to give

$$
\lambda_{p}(\bar{u})-\sigma \geqslant 1 / G_{0} M .
$$

Therefore we conclude

$$
\lambda_{r}[0]-\lambda_{s}[0]=\lambda_{p}(\bar{u})-\sigma \geqslant 1 / G_{0} M .
$$

Proof of Proposition (6.7). Assume that $Q(T)-Q(0) \leqslant 1 /(G M)^{2}$. We show that $\gamma_{r}$ intersects $\gamma_{s}$ in time [0,T/2]. By Proposition (5.2) this implies that either $r \in \mathscr{N}(T)$ or $s \in \mathscr{N}(T)$. Thus it suffices to show that $x_{s}(T / 2)-x_{r}(T / 2) \leqslant 0$. By (5.53),

$$
\begin{aligned}
\left|\lambda_{s}[T / 2]-\lambda_{s}[0]\right| & \leqslant G_{0}\left\{B_{s}+B_{s}^{-}+[Q(0)-Q(T)]^{1 / 2}\right\} \\
& \leqslant G_{0}\{\delta / M+\delta / M+1 / G M\} \leqslant \frac{3 G_{0} \delta}{M},
\end{aligned}
$$

where we applied Lemmas (6.10) and (6.11). Therefore we can use (5.42) and (5.44) to obtain

$$
\begin{aligned}
x_{s}(T / 2) & \leqslant x_{s}(0)+\int_{0}^{T / 2} \lambda_{s}(t) d t+\left(G_{0} h t_{J}\right)^{1 / 2} \\
& \leqslant x_{s}(0)+\lambda_{s}[0] T / 2+\left(3 \delta G_{0} / M\right) T / 2+\left(G_{0} h t_{J}\right)^{1 / 2} .
\end{aligned}
$$

Similarly,

$$
x_{r}(T / 2) \geqslant x_{r}(0)+\lambda_{r}[0] T / 2-\left(3 \delta G_{0} / M\right) T / 2-\left(G_{0} h t_{J}\right)^{1 / 2} .
$$


Subtracting (6.89) from (6.88) gives

$$
\begin{aligned}
x_{s}(T / 2)-x_{r}(T / 2) \leqslant & {\left[x_{s}(0)-x_{r}(0)\right]+\left\{\lambda_{s}[0]-\lambda_{r}[0]\right\} T / 2 } \\
& +2\left\{3 \delta G_{0} T / 2 M+\left(G_{0} h t_{J}\right)^{1 / 2}\right\} ;
\end{aligned}
$$

and by Lemmas (6.5) and (6.12), respectively,

$$
\left|x_{s}(0)-x_{r}(0)\right| \leqslant \varepsilon M / \delta, \quad \lambda_{s}[0]-\lambda_{r}[0] \leqslant-1 / G_{0} M .
$$

Therefore

$$
\begin{aligned}
x_{s}(T / 2)-x_{r}(T / 2) & \leqslant\left\{\frac{\varepsilon M}{\delta}+\frac{3 \delta G_{0} T}{M}+2\left(G_{0} h t_{J}\right)^{1 / 2}-\frac{T}{2 G_{0} M}\right\} \\
& =\left\{\frac{2 G_{0} M^{2} \varepsilon}{\delta T}+6 G_{0}^{2} \delta+\frac{4 G_{0} M\left(G_{0} h t_{J}\right)^{1 / 2}}{T}-1\right\} \frac{T}{2 G_{0} M} .
\end{aligned}
$$

By (6.28) and (6.22), (6.23),

$$
\frac{2 G_{0} M^{2} \varepsilon}{\delta T}=\frac{2 G_{0} M^{2} \varepsilon}{\delta \varepsilon G^{2} M^{2}}=\frac{2 G_{0}}{\delta G^{2}}=2 \delta^{3} G_{0}<\frac{1}{3} .
$$

By (6.22)

$$
6 G_{0}^{2} \delta<1 / 3
$$

By (6.28) and (6.33),

$$
\frac{4 G_{0} M\left(G_{0} h t_{J}\right)^{1 / 2}}{T} \leqslant \frac{4 \sqrt{2} M G_{0}^{3 / 2} h^{1 / 2}}{T^{1 / 2}}<\left(\frac{4 \sqrt{2} M G_{0}^{3 / 2}}{T^{1 / 2}}\right)\left(\frac{T^{1 / 2}}{12 \sqrt{2} M G_{0}^{3 / 2}}\right)<\frac{1}{3} .
$$

Finally, putting (6.92)-(6.94) into (6.91) yields

$$
x_{s}(T / 2)-x_{r}(T / 2)<0 \text {. }
$$

This completes the proof of Proposition (6.7), and hence also completes the proof of Theorem (6.3). The proof of Theorem (6.3) also applies to the case of periodic data, in which case \|\|$_{L^{1}},\|\|_{S}$ and $Q(t)$ are defined on each period.

\section{REFERENCES}

1. I-Liang Chern, On the decay of a strong wave for hyperbolic conservation laws in one space dimension, Thesis, Courant Institute, New York Univ., 1982.

2. C. Courant and K. O. Friedrichs, Supersonic flow and shock waves, Springer-Verlag, Berlin and New York, 1948.

3. C. Dafermos, Application of the invariance principle for compact processes. II. Asymptotic behavior of solutions of a hyperbolic conservation law, J. Differential Equations 11 (1972), 416-424.

4. R. DiPerna, Decay and asymptotic behavior of solutions to nonlinear hyperbolic systems of conservation laws, Indiana Univ. Math. J. 24 (1975), 1047-1071.

5. Decay of solutions of hyperbolic systems of conservation laws with a convex extension, Arch. Rational Mech. Anal. 69 (1977), 1-46.

6. J. Glimm, Solutions in the large for nonlinear hyperbolic systems of equations, Comm. Pure Appl. Math. 18 (1965), 695-715.

7. J. Glimm and P. Lax, Decay of solutions of systems of nonlinear hyperbolic conservation laws, Mem. Amer. Math. Soc. No. 101 (1970). 
8. B. Keyfitz, Solutions with shocks: an example of an $L^{1}$-contractive semigroup, Comm. Pure Appl. Math. 24 (1971), 125-132.

9. P. Lax, Hyperbolic systems of conservation laws, II. Comm. Pure Appl. Math. 10 (1957), 537-566.

10. T. P. Liu, Asymptotic behavior of solutions of general system of nonlinear hyperbolic conservation laws, Indiana Univ. J. 27 (1978), 211-253.

11. __ Admissible solutions of hyperbolic conservation laws, Mem. Amer. Math. Soc. No. 240 (1981).

12. Decay to $N$-waves of solutions of general system of nonlinear hyperbolic conservation laws, Comm. Pure Appl. Math. 30 (1977), 585-610.

13. L__ Large-time behavior of solutions of initial and initial-boundary value problem of general system of hyperbolic conservation laws, Comm. Math. Phys. 55 (1977), 163-177.

14. L Linear and nonlinear large-time behaviors of solutions of hyperbolic conservation laws, Comm. Pure Appl. Math. 30 (1977), 767-796.

15. __ The deterministic version of the Glimm scheme, Comm. Math. Phys. 57 (1977), 135-148.

16. L. Luskin and J. B. Temple, The existence of a global weak solution to the nonlinear Waterhammer problem, Comm. Pure Appl. Math. 35 (1982), 697-735.

17. J. A. Smoller, Shock waves and reaction-diffusion equations, Springer-Verlag, New York, 1980.

18. T. B. Temple, Global solution of the Cauchy problem for a class of $2 \times 2$ nonstrictly hyperbolic conservation laws, Adv. in Appl. Math. 3 (1982), 335-375.

19. Solutions in the large for the nonlinear hyperbolic conservation laws of gas dynamics, J. Differential Equations 41 (1981), 96-161.

20. Systems of conservaton laws with coinciding shock and rarefaction curves, Contemp. Math. 17 (1983), 143-151.

21. Systems of conservation laws with invariant submanifolds, Trans. Amer. Math. Soc. 280 (1983), 781-795.

22. , No $L^{1}$-contractive metrics for systems of conservation laws, Trans. Amer. Math. Soc. 288 (1985),

23. __ On supnorm bounds in Glimm's method, Mathematics Research Center Technical Summary Reports, \#2855, University of Wisconsin, Madison.

Mathematics Research Center, University of Wisconsin, Madison, Wisconsin 53706

Current address: Department of Mathematics, University of California, Davis, California 95616 